\title{
Supporting Information: Steric Interactions Impact Vibronic and Vibrational Coherences in Perylenediimide Cyclophanes
}

\author{
Jonathan D. Schultz, Adam F. Coleman, Aritra Mandal, Jae Yoon Shin, Mark A. Ratner, \\ Ryan M. Young, ${ }^{*}$ and Michael R. Wasielewski* \\ Department of Chemistry and Institute for Sustainability and Energy at Northwestern, \\ Northwestern University, Evanston, IL 60208-3113, USA \\ *Correspondence to: $\underline{\text { m-wasielewski@northwestern.edu, ryan.young @ northwestern.edu }}$
}

\section{Table of Contents}

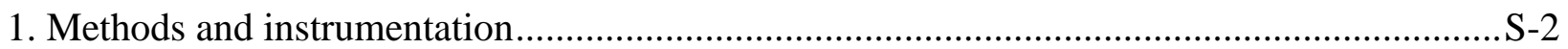

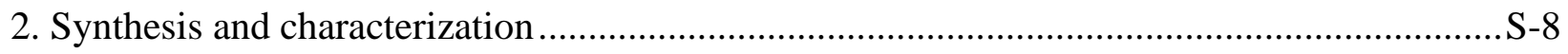

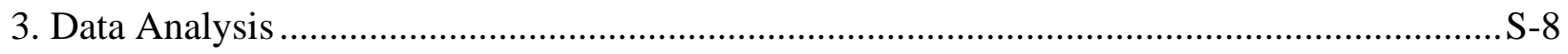

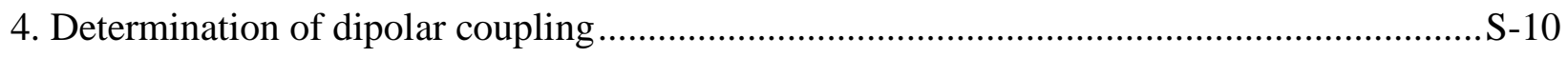

5. Additional two-dimensional electronic spectroscopy .................................................

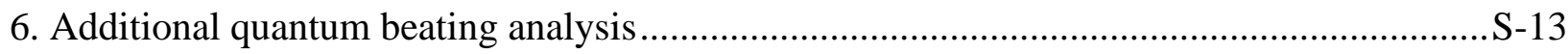

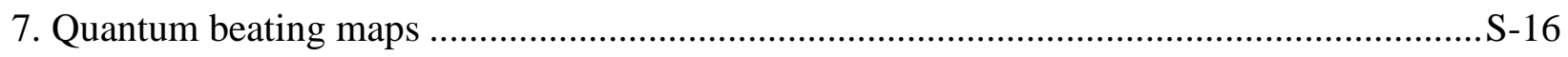

8. Impact of resonance condition and high-frequency coherences ......................................S-17

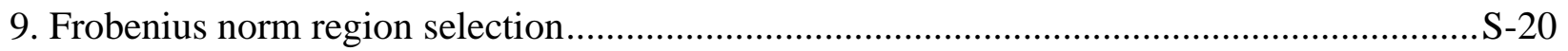

10. Characterization of influence from the dynamic Stokes shift.......................................S-21

11. Additional femtosecond stimulated Raman spectroscopy data ......................................S-23

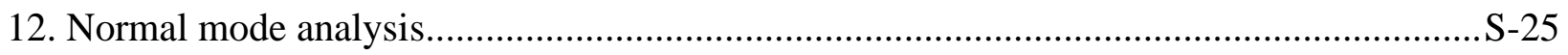

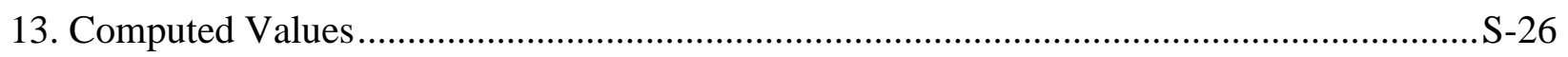

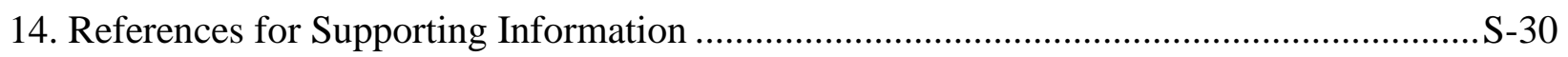




\section{Methods and instrumentation}

\subsection{Synthesis and characterization}

Synthetic procedures for the 4-t-butyltetraphenoxy bay-substituted perylenediimide paracyclophane ( $\boldsymbol{p}$-PDI) and the monomeric reference (PDI-Ref) compound can be found in previous literature. $^{1-2}$

\section{Synthesis of meta-cyclophane ( $m$-PDI):}

1,6,7,12-tetra(4-t-butylphenoxy)perylene-3,4:9,10-tetracarboxylic acid dianhydride (100mg, 0.1mmol), 1,3-bis(aminomethyl)benzene $(1.4 \mathrm{~mL}$ of a solution of $0.1 \mathrm{~mL}$ diamine in 10mL toluene, $0.1 \mathrm{mmol})$, imidazole $(1 \mathrm{~g}, 15 \mathrm{mmol})$, pyridine $(10 \mathrm{~mL}, 124 \mathrm{mmol})$, and toluene (200 mL) were mixed. The mixture was heated to $115^{\circ} \mathrm{C}$ for 18 hours. After cooling to room temperature, $250 \mathrm{~mL}$ of $2 \mathrm{M} \mathrm{HCl}$ was added. The organic layer was collected and washed with water. The remaining aqueous layer was extracted with DCM, and the organic layer was collected. The solvent was removed from the combined organic layers under reduced pressure. The crude product was purified via column chromatography (DCM, silica) and HPLC to yield $23 \mathrm{mg}$ of $m$-PDI (6\%).

${ }^{1} \mathrm{H}-\mathrm{NMR}(600 \mathrm{MHz}, 363 \mathrm{~K}, \mathrm{C} 2 \mathrm{D} 2 \mathrm{Cl4}): \delta=8.08(\mathrm{~s}, 8 \mathrm{H}), 7.45(\mathrm{~d}, \mathrm{~J}=7.74 \mathrm{~Hz}, 4 \mathrm{H}), 7.32(\mathrm{t}, \mathrm{J}=7.74$ Hz, 2H), 7.28 (bs, 2H), 7.23 (d, J=8.22 Hz, 16H), 6.73 (bs, 16H), 5.24 (s, 8H), 1.33 (s, 72H) ppm. HRMS (APPI, positive mode, DCM): m/z: calculated for $\mathrm{C}_{144} \mathrm{H}_{129} \mathrm{~N}_{4} \mathrm{O}_{16}: 2169.9398$ [M+H] ${ }^{+}$, found: 2169.9349.

\subsection{Linear electronic spectroscopy}

Steady-state UV/Visible absorption spectra for room temperature solutions of each compound were collected by a Shimadzu UV-1800 spectrophotometer. Solutions of each analyte 
in tetrahydrofuran (THF, dried using a Glass Contour solvent system) were prepared with maximum optical densities of 0.6 in glass cuvettes with a $1 \mathrm{~mm}$ pathlength.

\subsection{Two-dimensional electronic spectroscopy (2DES)}

2DES experiments were conducted using an experimental apparatus previously described. ${ }^{3}$ Briefly, the $1040 \mathrm{~nm}$ fundamental output of a Yb:KGW regenerative amplifier system at $100 \mathrm{kHz}$ (Spirit 1040-4, Spectra Physics, Inc.) was used to generate femtosecond pulses in a noncollinear optical parametric amplifier (Spirit-NOPA, Light Conversion Inc.) to be used for the pump. These pulses were frequency dispersed into a tellurium dioxide acousto-optic modulator (AOM)-based pulse shaper to create two time-ordered pulses from each input pulse and to correct for dispersion. ${ }^{4}$ For the experiment discussed in section 8, a quartz AOM was employed. The experiments conducted for this work employed pump pulses centered at $\sim 17550 \mathrm{~cm}^{-1}(\sim 570 \mathrm{~nm}$, Figure S1). These pulses were $\sim 28$ fs in duration, as characterized by second harmonic generation frequencyresolved optical gating (SHG-FROG, Figure S2). The two time-ordered pump pulses generated by the pulse shaper with coherence time delay $\left(\tau_{l}\right)$ were focused at the sample with dispersioncorrected white light (WL) probe pulses spanning 500-1000 nm. 2DES experiments were conducted in the pump-probe geometry using a commercial 2D spectrometer (2DQuickVIS, Phasetech Spectroscopy, Inc.). The pump and probe were parallel in polarization and the pump energy at the sample was $\sim 12 \mathrm{~nJ} /$ pulse. Data were collected in the rotating frame with a rotation frequency of $15500 \mathrm{~cm}^{-1}$ and undersampled from 0 to $300 \mathrm{fs}$ with a 4 fs step size. The data with respect to this time dimension were Fourier transformed in post-processing (vide infra) to produce the $\omega_{1}$ axis of the $2 \mathrm{D}$ spectrum, which thereby reflects energies provided to the chemical system via the pump pulses. For each $\tau_{1}$, spectra that reflect the third order signal heterodyned with the 
probe are collected to create the $\omega_{3}$ dimension. The delay between the second pump and the probe pulses (the population time $\tau_{2}$ ) is controlled by a digital delay stage. Four-frame phase cycling was used to remove transient absorption and pump scatter background signals. Data were averaged between 1000 and 5000 laser cycles for each 2D spectrum depending on the sample optical density. To evaluate quantum beating signatures, a series of spectra were collected as a function of $\tau_{2}$. This delay was scanned from -100 to $1500 \mathrm{fs}$ with a timestep of $10 \mathrm{fs}$, which yields a frequency resolution of $\sim 24 \mathrm{~cm}^{-1}$ (vide infra). Samples for transient experiments were prepared as described above. Numerous experiments were conducted on different days with different solvent aliquots to ensure the results were not correlated to laser fluctuations or solvent impurities (Figures S9-11). The scans presented in the main text were averaged over 1000 laser cycles and used sample optical densities of $\sim 0.25, \sim 0.45$, and $\sim 0.28$ for PDI-Ref, $\boldsymbol{p}$-PDI, and $\boldsymbol{m}$-PDI, respectively.

\subsection{Laser pulse characterization}

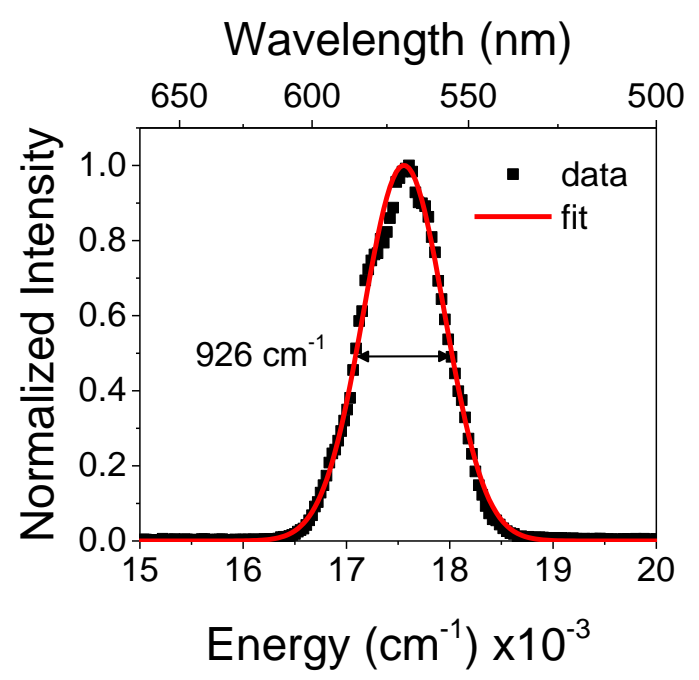

Figure S1. A typical pump pulse spectrum. Fitting to a single Gaussian yields a FWHM of $926 \mathrm{~cm}^{-1}$.

Figure S1 shows a typical chirp-corrected pulse spectrum, centered at $17540 \mathrm{~cm}^{-1}(570$ $\mathrm{nm}$ ), spanning 16500 to $18600 \mathrm{~cm}^{-1}$ with a $~ 926 \mathrm{~cm}^{-1}$ full-width at half-maximum (FWHM). Group 
velocity and third-order dispersions were compensated for using the pulse shaper. A commercial second-harmonic generation frequency resolved optical gating (SHG-FROG) instrument (Mesa Photonics Inc.) was used to visualize the pump pulses in the time domain and determine optimal chirp correction parameters. Example SHG-FROG traces are provided in Figure S2A and B. The measured pulse width of the 2DES pump pulse was $\sim 28 \mathrm{fs}$.
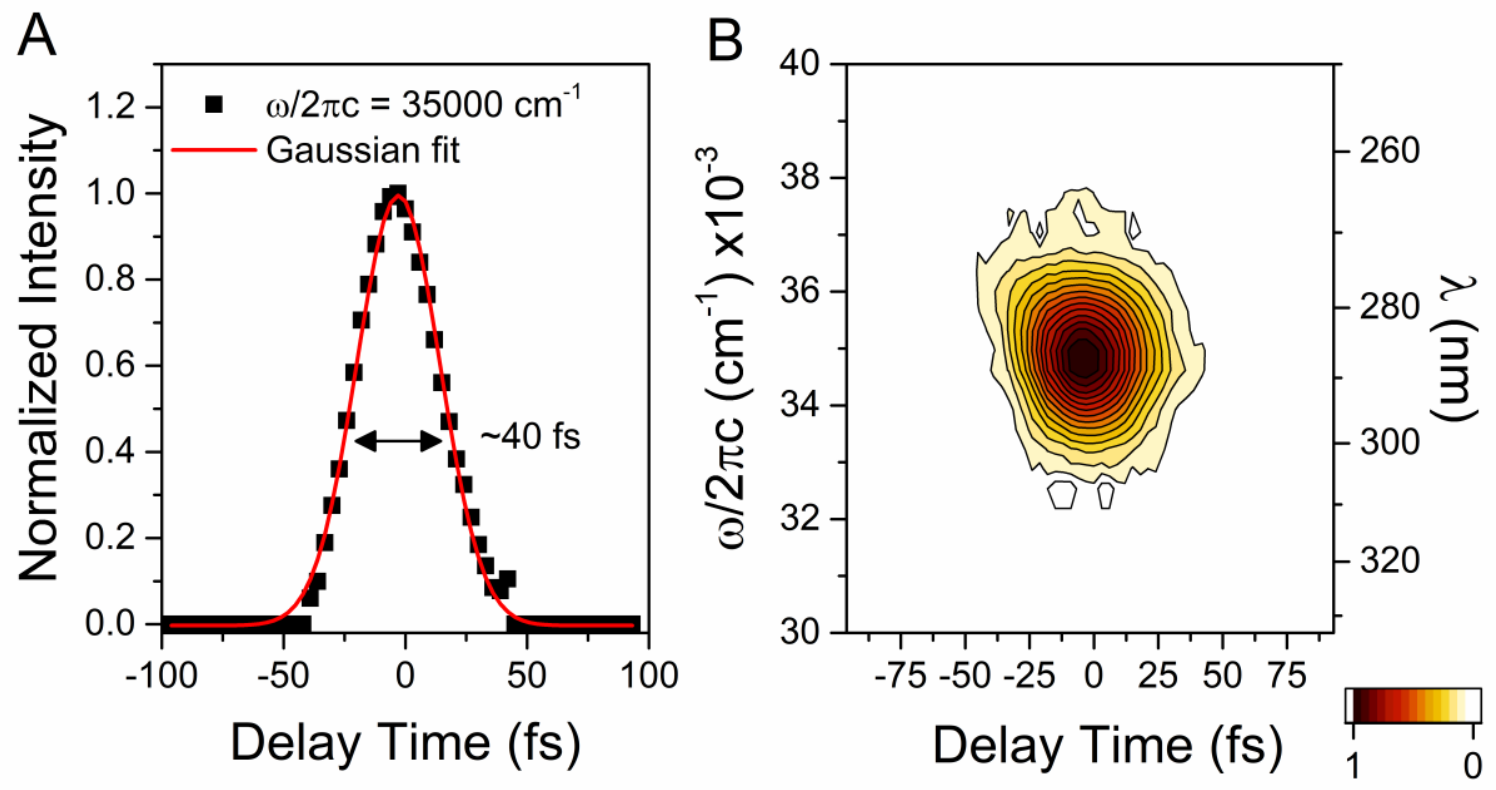

Figure S2. (A) SHG-FROG trace of the typical pump pulse employed in these experiments and (B) a single time-domain slice of the FROG trace overlaid with a Gaussian fit (FWHM $=\sim 40$ fs). The average pulse duration determined via six FROG traces was $\sim 28$ fs.

\subsection{Instrument response characterization}

Polarization-gated frequency resolved optical gating (PG-FROG) measurements were performed to determine the frequency-dependent instrument response function between the pump and probe pulses under similar experimental conditions to those shown in the main text. These measurements were conducted with the dispersion-corrected pump pulse as the gate and the whitelight supercontinuum as the probe in the same beam geometry utilized for the 2DES experiments. 
For PG-FROG measurements, a polarizer was used to minimize the probe intensity on the spectrometer and the polarization of the pump pulse was rotated $45^{\circ}$ relative to that of the probe. Both beams were spatially and temporally overlapped in a $1 \mathrm{~mm}$ cuvette filled with neat DCM solvent. Figure S3 shows the PG-FROG signal (pre-time zero signal subtracted) obtained for the GSB and ESA regions of interest to the molecular systems in this study.

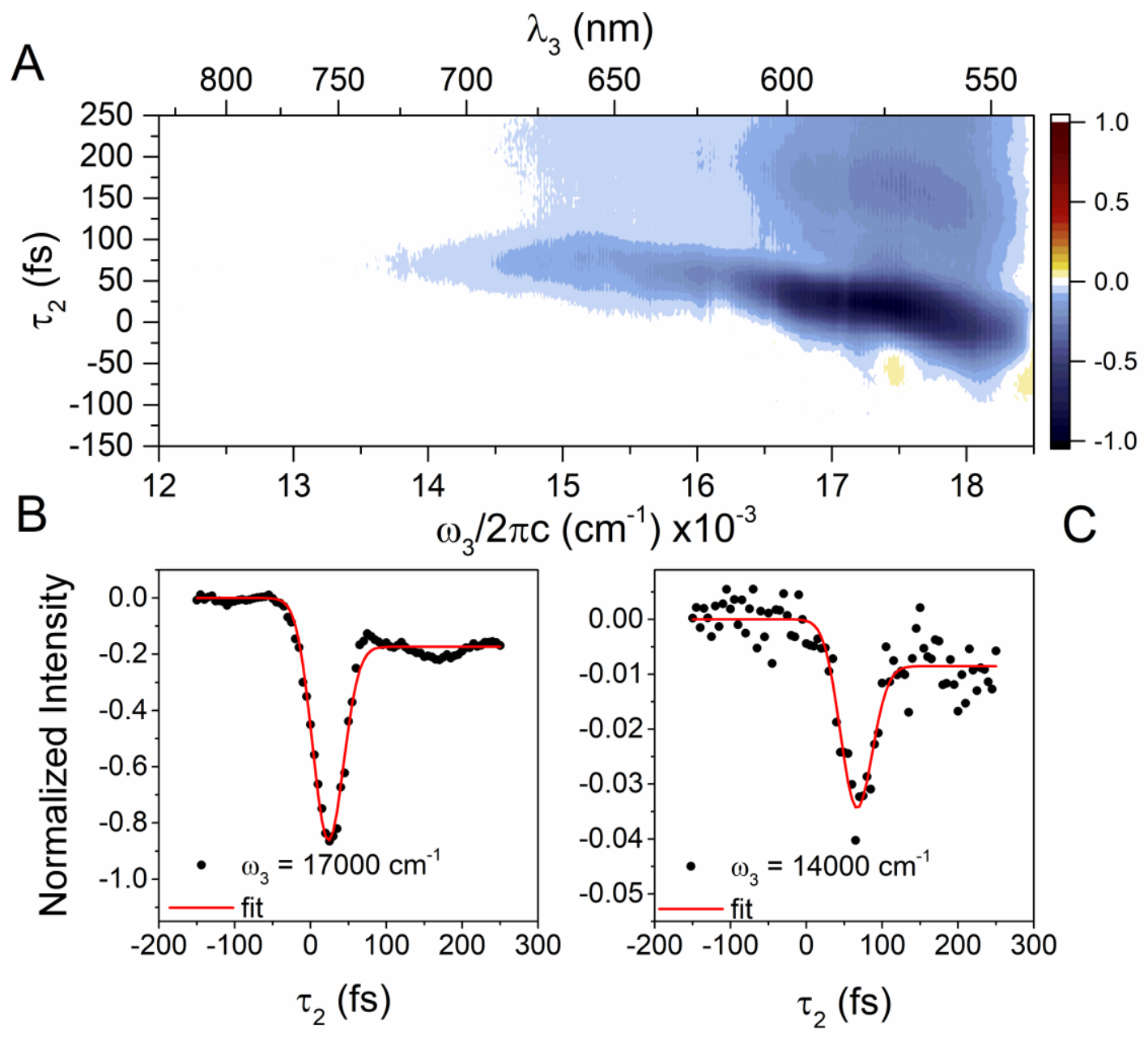

Figure S3. (A) OKE signal across the ESA region of the compounds in this work and a time-domain slices at (B) $\omega_{3} \sim 17000 \mathrm{~cm}^{-1}$ and (C) $\omega_{3} \sim 14000 \mathrm{~cm}^{-1}$ of the OKE trace overlaid with a Gaussian-shelf convolution fit (FWHM $\sim 48$ and 49 fs respectively). 


\subsection{Femtosecond Stimulated Raman Spectroscopy (FSRS)}

FSRS experiments were preformed using a previously described laser system. ${ }^{5}$ Briefly, the setup operates using the $800 \mathrm{~nm}, 35$ fs fundamental output of a regeneratively amplified $1 \mathrm{kHz}$ Ti:Sapphire laser system (Spitfire Pro XP, Spectra Physics, Inc.). For experiments probing the ground state, a $600 \mathrm{~nm}$ Raman pump generated via a second harmonic bandwidth compressor pumping an optical parametric amplifier (SHBC/TOPAS-400, Light Conversion, LLC) was used. The broadband WL Raman probe is produced by focusing the fundamental $800 \mathrm{~nm}$ light into an $\mathrm{H}_{2} \mathrm{O} / \mathrm{D}_{2} \mathrm{O}$ mixture. The Raman pump was $2 \mu \mathrm{J} /$ pulse at the sample and parallel in polarization with the probe for the measurements described here. Data were processed into Raman gain by dividing the heterodyned signal and probe spectrum with the Raman pump incident by the probe spectrum without the pump present. For excited-state experiments, a tunable actinic pump (573 nm, 1 $\mu \mathrm{J} /$ pulse) is generated via an optical parametric amplifier (TOPAS-C, Light Conversion, LLC) and used in conjunction with a $710 \mathrm{~nm}$ Raman pump. Polarization of the actinic pump was set at $54.7^{\circ}$ (magic angle) relative to the Raman pump/probe to negate rotational effects. The timing between the actinic pump and Raman pump/probe pair is controlled with a motorized delay stage. Samples for these experiments were prepared as described above, but with slightly higher concentrations (less than $200 \mu \mathrm{M}$ ) and in cuvettes with a $2 \mathrm{~mm}$ pathlength.

\subsection{Computational details}

All DFT calculations were performed using the Q-Chem 5.0 software package at the level of B3LYP/6-31G* in vacuo. To lower the computational cost, the tert-butyl groups of the phenoxy substituents were substituted with methyl groups. The fully optimized geometry of PDI-Ref was confirmed by ensuring no imaginary frequencies were present in the final frequency output. To remove an imaginary frequency, we scaled the system geometry by an arbitrary percentage of the 
imaginary mode motion, re-conducted the geometry optimization and frequency analysis, and iterated the process further if the negative frequency remained.

\section{Synthesis and characterization}

${ }^{1} \mathrm{H}$ NMR was collected at $363 \mathrm{~K}$ on a Bruker Avance III $600 \mathrm{MHz}$ system. Mass spectrometry was performed on a Bruker IMPACT II to generate high-resolution HR-APPI-MS spectra at the Integrated Molecular Structure Education and Research Center (IMSERC) at Northwestern University. All solvents and reagents were purchased from commercial suppliers and used without purification. Column chromatography was performed using silica gel from Macherey-Nagel and HPLC was performed on a Shimadzu HPLC.

\section{Data Analysis}

\subsection{DES Population analysis}

To isolate coherent dynamics in these studies, we fit population dynamics at each $\left(\omega_{1}, \omega_{3}\right)$ coordinate to an offset exponential function following the form:

$$
S\left(\omega_{1}, \omega_{3}, t_{2}\right)=S_{0}+A_{1} e^{-t_{2} / \tau_{1}}+A_{2} e^{-t_{2} / \tau_{2}}
$$

where $S_{0}$ is the vertical offset, $A_{i}$ and $\tau_{i}$ are the amplitude and time constant of component $i$, respectively. Only waiting times after $100 \mathrm{fs}$ were considered so as to avoid influence from pulse overlap effects. One exponential decay was used to remove rapid population dynamics ( $<300 \mathrm{fs})$ likely from inertial solvent motion ${ }^{6-7}$ as well as interexciton relaxation in the dimers. ${ }^{8} \mathrm{~A}$ second exponential function was included to remove a longer time component ( 1-2 ps) likely attributable to vibrational cooling owing to weak excitation of the higher vibronic bands due to the large pump bandwidth. Examples fits for the higher energy $S_{n} \leftarrow S_{1}$ transition for each compound are shown in Figure 3A-C of the main text. Figure S4 shows additional fits of Equation (S1) for the ground- 
state bleach (GSB)/stimulated emission (SE) and excited-state absorption (ESA) features of $\boldsymbol{p}$-PDI and the resultant power spectra.
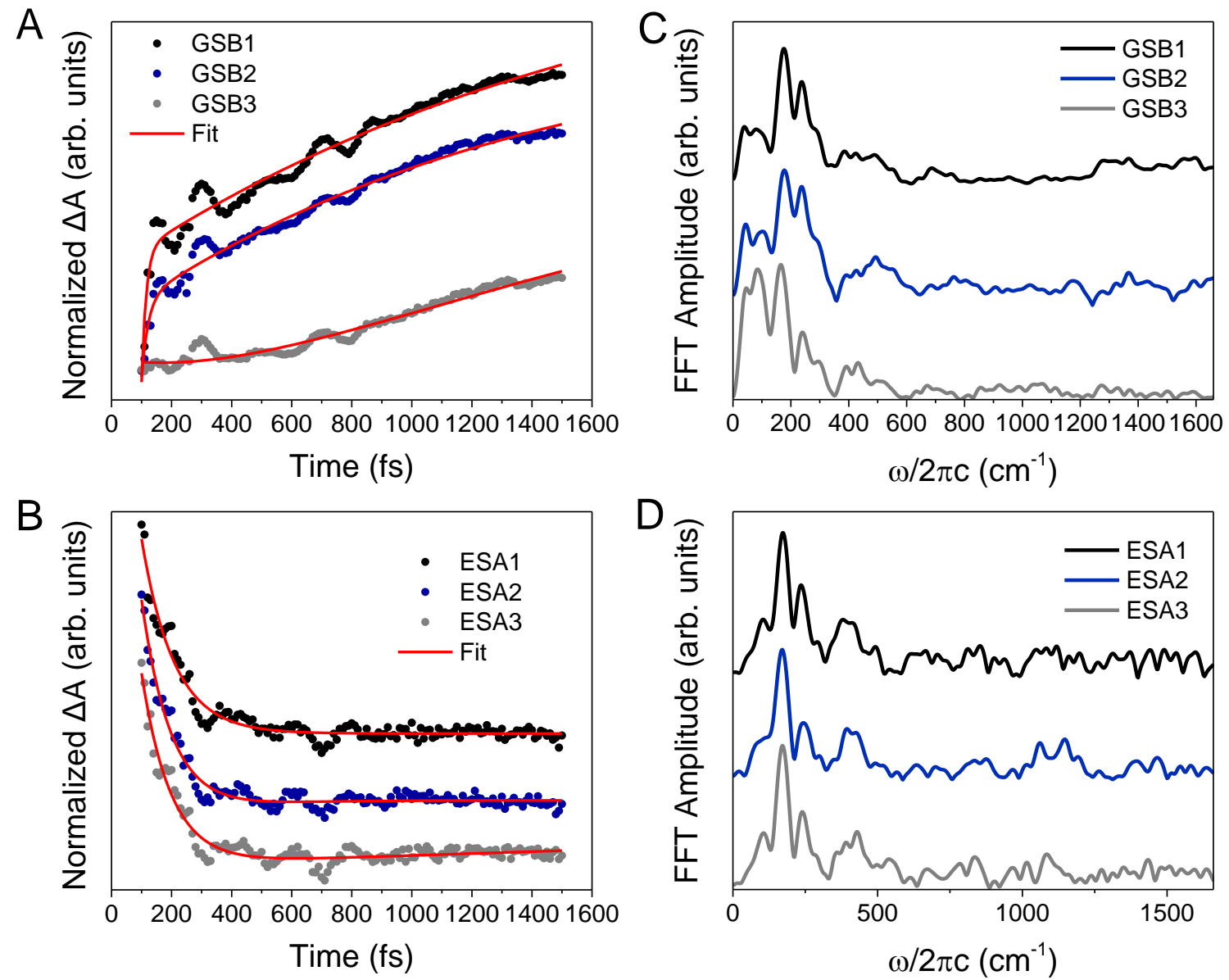

Figure S4. Representative (A and B) bi-exponential fits and (C and D) resultant power spectra for single $\left(\omega_{1}, \omega_{3}\right)$ coordinates on the GSB/SE (top) and ESA (bottom) features for $\boldsymbol{p}$-PDI.

\subsection{Fast Fourier transform analysis}

Generation of 2DES spectra requires Fourier transformation of the data with respect to the coherence time delay $\tau_{1}$. This procedure involves multiplication of the data by a Hanning window function to bring the end of each trace smoothly to zero. The data are then zero-padded to a vector length of $2^{8}$ prior to fast Fourier transformation (FFT). After isolation from population kinetics, Fourier analysis of quantum beating traces with respect to the population time follows a similar 
procedure for that of the coherence time delay. However, these data are zero-padded to a length of $2^{10}$. Since data between 0 and near-100 fs are truncated, zeros are input as corresponding placeholders to ensure no systematic frequency shifts. The unpadded data traces range from $\sim 100$ to $1500 \mathrm{fs}$ with a timestep of $10 \mathrm{fs}$, which yields a frequency resolution of $\sim 24 \mathrm{~cm}^{-1}$ in the Fourier power spectra.

\section{Determination of dipolar coupling}

Simulations using a previously described Frenkel-Holstein Hamiltonian formalism ${ }^{9}$ were conducted to estimate the dipolar coupling in these dimeric systems. Spectral linewidths were imposed by applying a Gaussian windowing function to the linear response and the absorption spectrum was obtained via a Fast Fourier transform. In these simulations, we included the primary vibration noted from the vibronic structure in the experimental absorption spectra of frequency $\sim 1250 \mathrm{~cm}^{-1}$. We first fit the monomer spectrum to extract a Huang-Rhys parameter of $\sim 0.56$. Employing these parameters in the dimer absorption spectra simulations, we calculated dipolar coupling values of $\sim 180$ and $\sim 270 \mathrm{~cm}^{-1}$ for $\boldsymbol{p}$-PDI and $\boldsymbol{m}$-PDI, respectively. The results of these simulations are shown in comparison to the experimental absorption spectra in Figure S5. 


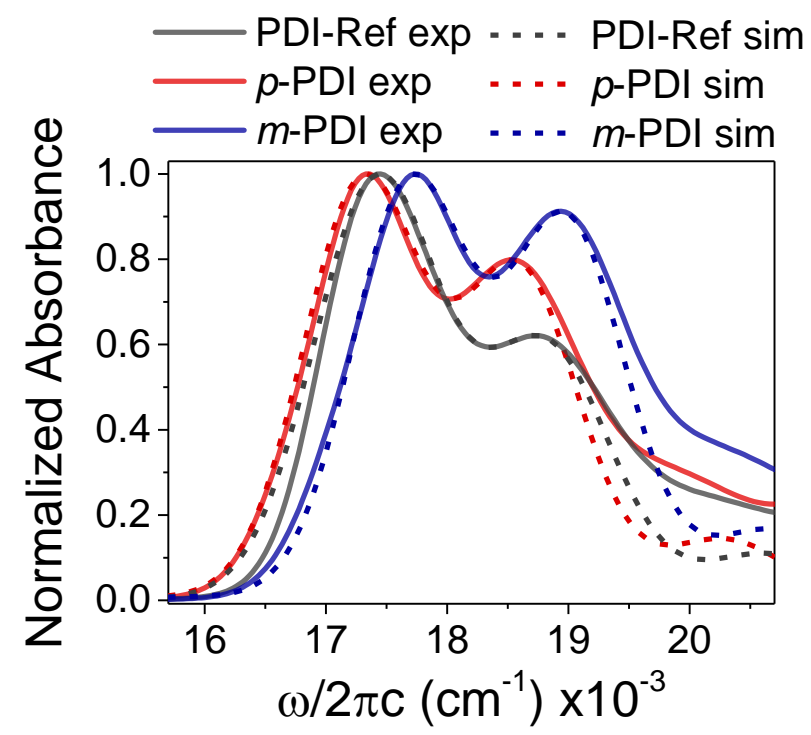

Figure S5. Experimental (solid) and simulated (dashed) absorption spectra for PDI-Ref, $\boldsymbol{p}$-PDI, and $\boldsymbol{m}$-PDI.

\section{Additional two-dimensional electronic spectroscopy}

Figures S6-S8 illustrate 2D spectra for PDI-Ref, $\boldsymbol{p}$-PDI, and $\boldsymbol{m}$-PDI as a function of the waiting time across the time range of interest to this work. For clarity, all 2DES spectra shown in this manuscript were smoothed with an $11^{\text {th }}$ order moving average. Contours near zero signal magnitudes are suppressed for clarity. Figure S8 illustrates that $\boldsymbol{m}$-PDI undergoes symmetrybreaking charge transfer $\left(\omega_{1}=17500 \mathrm{~cm}^{-1}, \omega_{3}=12800 \mathrm{~cm}^{-1}\right)$, but to a negligible amount $(<5 \%)$ within the temporal window of interest to this work (1.5 ps). 


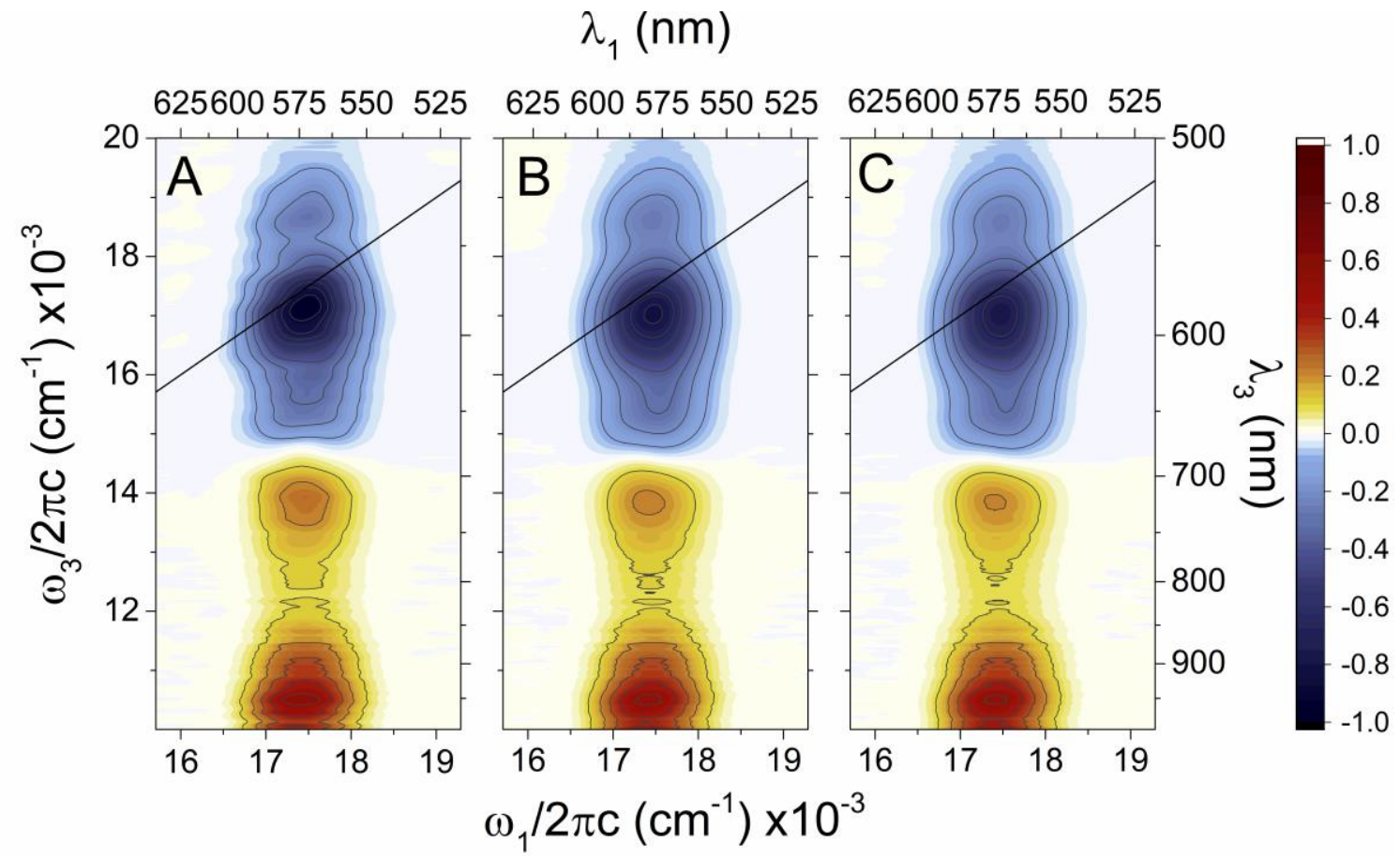

Figure S6. Absorptive 2DES spectra for PDI-Ref at waiting times of (A) 100, (B) 500, and (C) $1500 \mathrm{fs}$ obtained in THF at $295 \mathrm{~K}$ and normalized to absolute maximum signal at $100 \mathrm{fs}$.

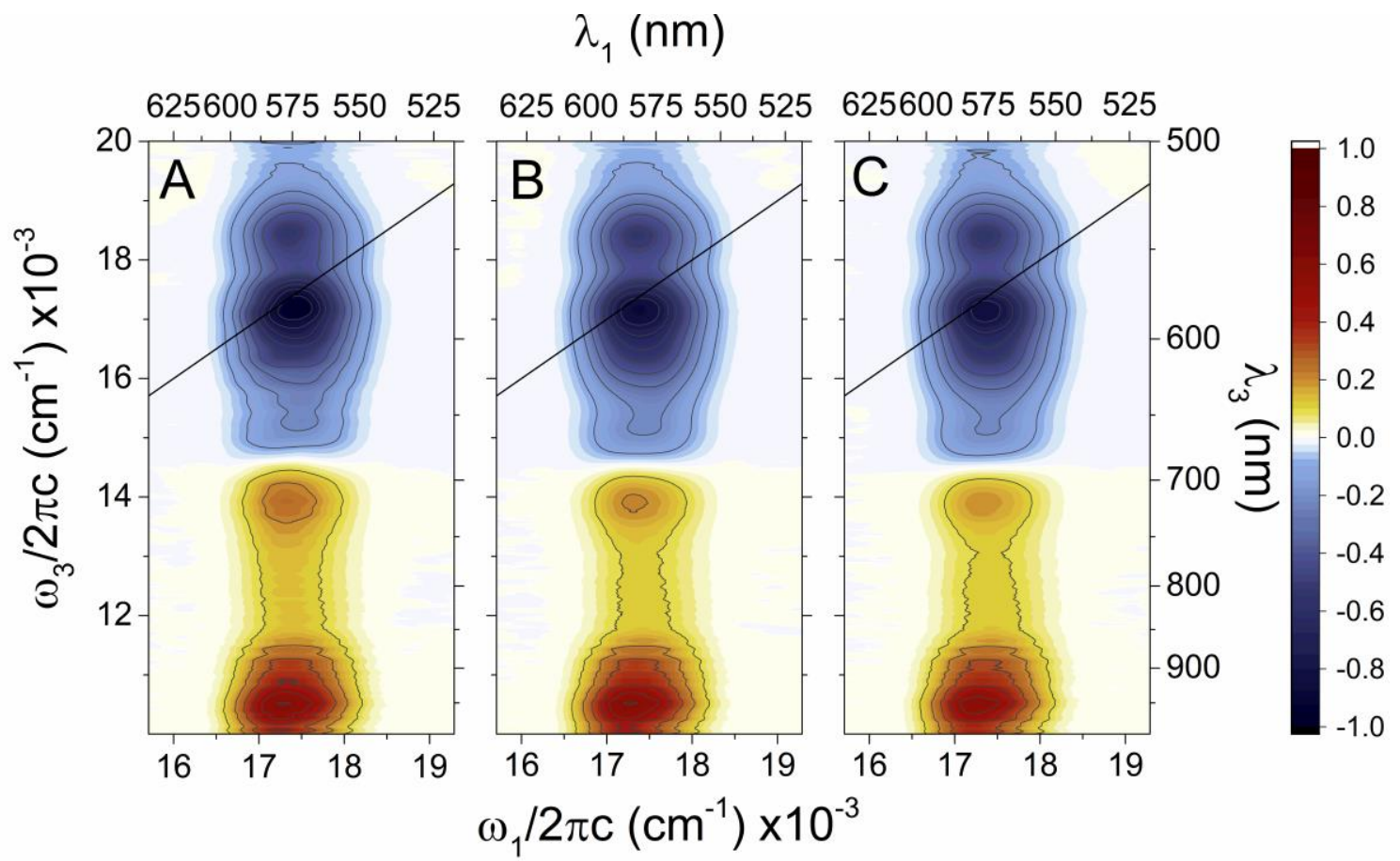

Figure S7. Absorptive 2DES spectra for p-PDI at waiting times of (A) 100, (B) 500, and (C) $1500 \mathrm{fs}$ obtained in THF at $295 \mathrm{~K}$ and normalized to absolute maximum signal at $100 \mathrm{fs}$. 


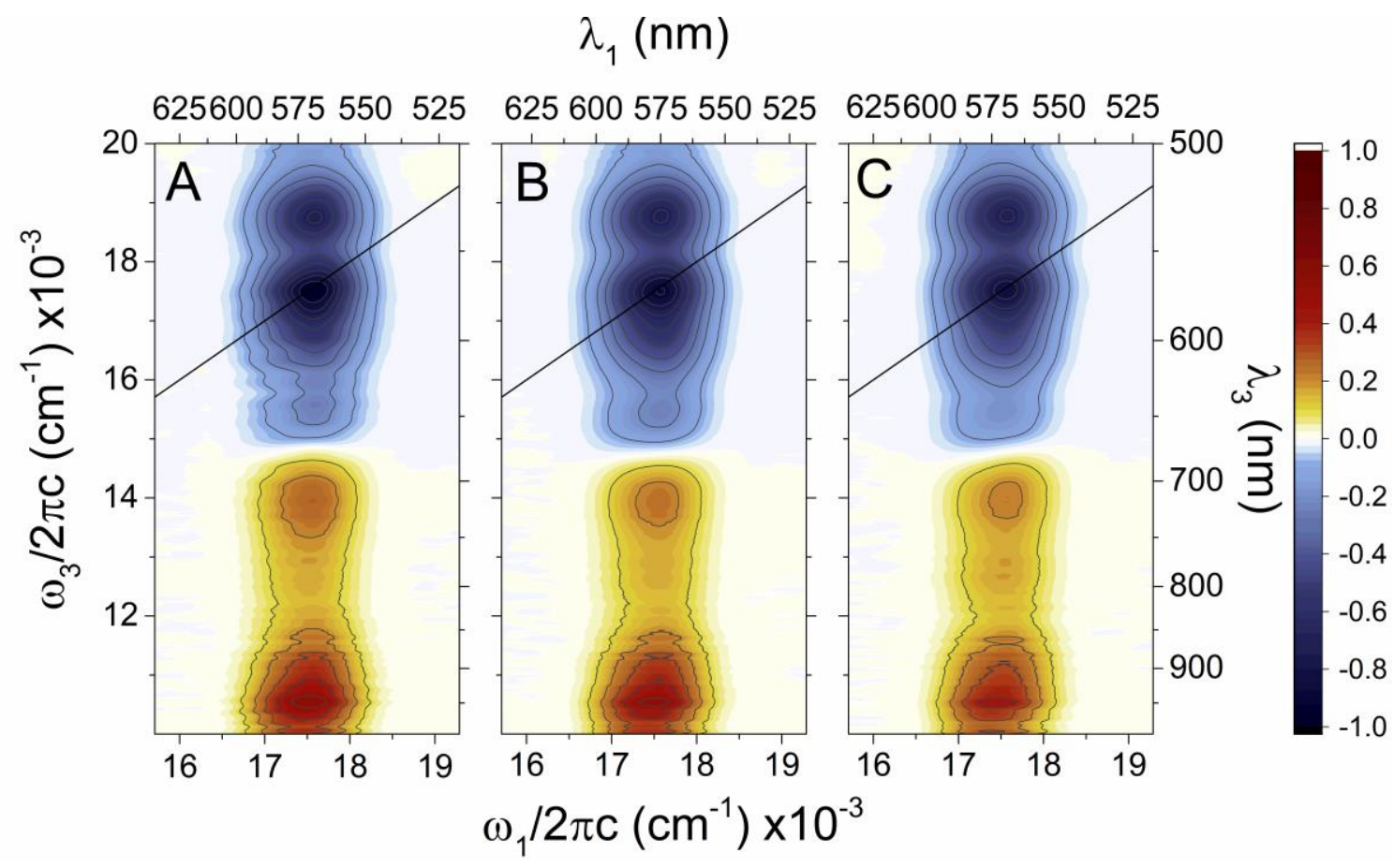

Figure S8. Absorptive 2DES spectra for $\boldsymbol{m}$-PDI at waiting times of (A) 100, (B) 500, and (C) $1500 \mathrm{fs}$ obtained in THF at $295 \mathrm{~K}$ and normalized to absolute maximum signal at $100 \mathrm{fs}$.

\section{Additional quantum beating analysis}

To confirm the quantum beating signatures shown in Figures 3 and 4 were not impacted by laser fluctuations and/or solvent impurities, we conducted these experiments numerous times on different days with different solvent aliquots and laser alignments. A compilation of the replicate power spectra for each compound is shown in Figures S9-11. The data shown in the main text are from scan 1. Furthermore, Figure S12 illustrates the results from a control measurement on neat-THF using the same experimental parameters and post-processing. 

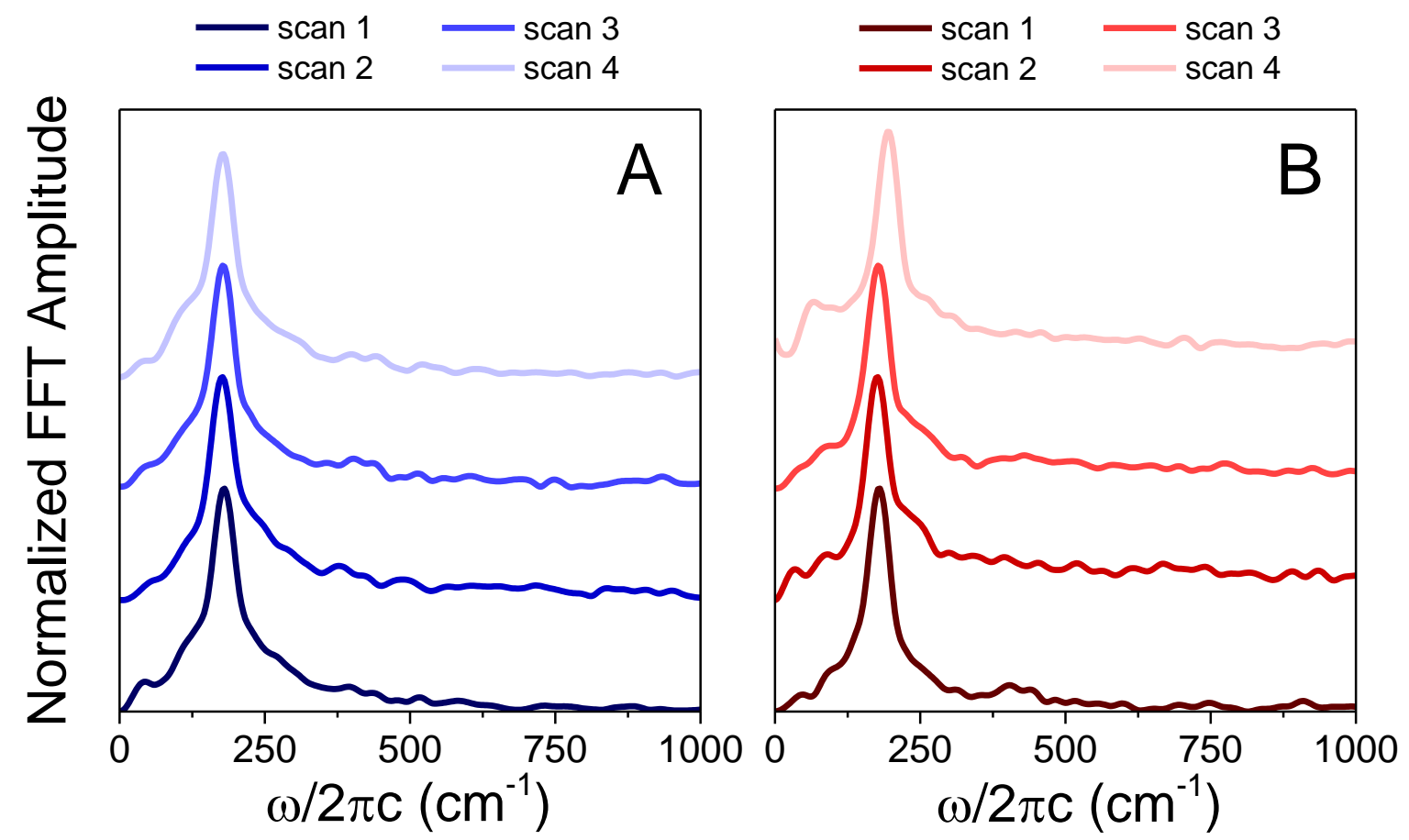

Figure S9. Normalized replicate Frobenius norms calculated across the PDI-Ref (A) GSB/SE and (B) ESA spectral features.
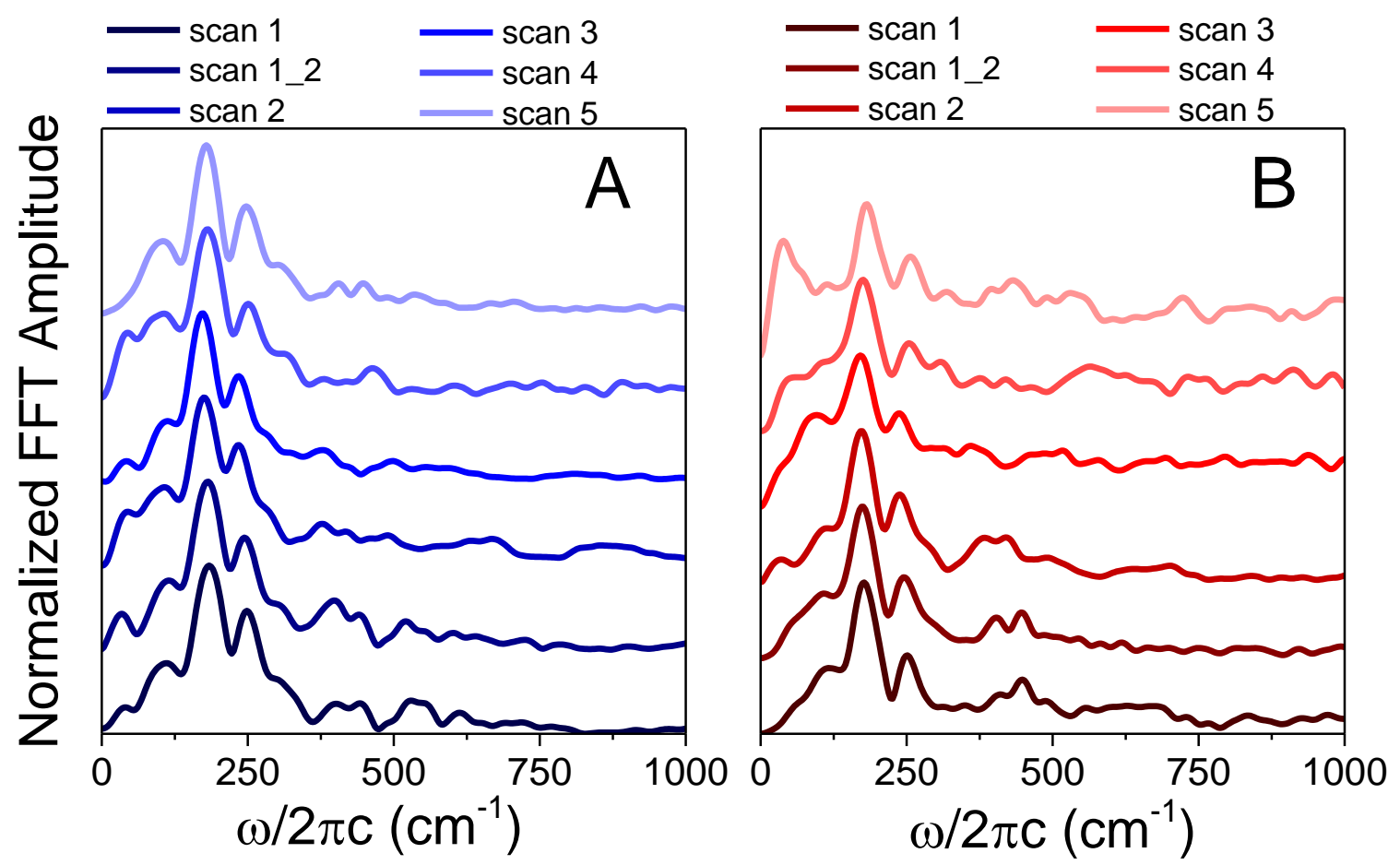

Figure S10. Normalized replicate Frobenius norms calculated across the p-PDI (A) GSB/SE and (B) ESA spectral features. All scans were collected on separate days, except scans 1 and 1_2 which were performed on the same day, but with different sample batches. 

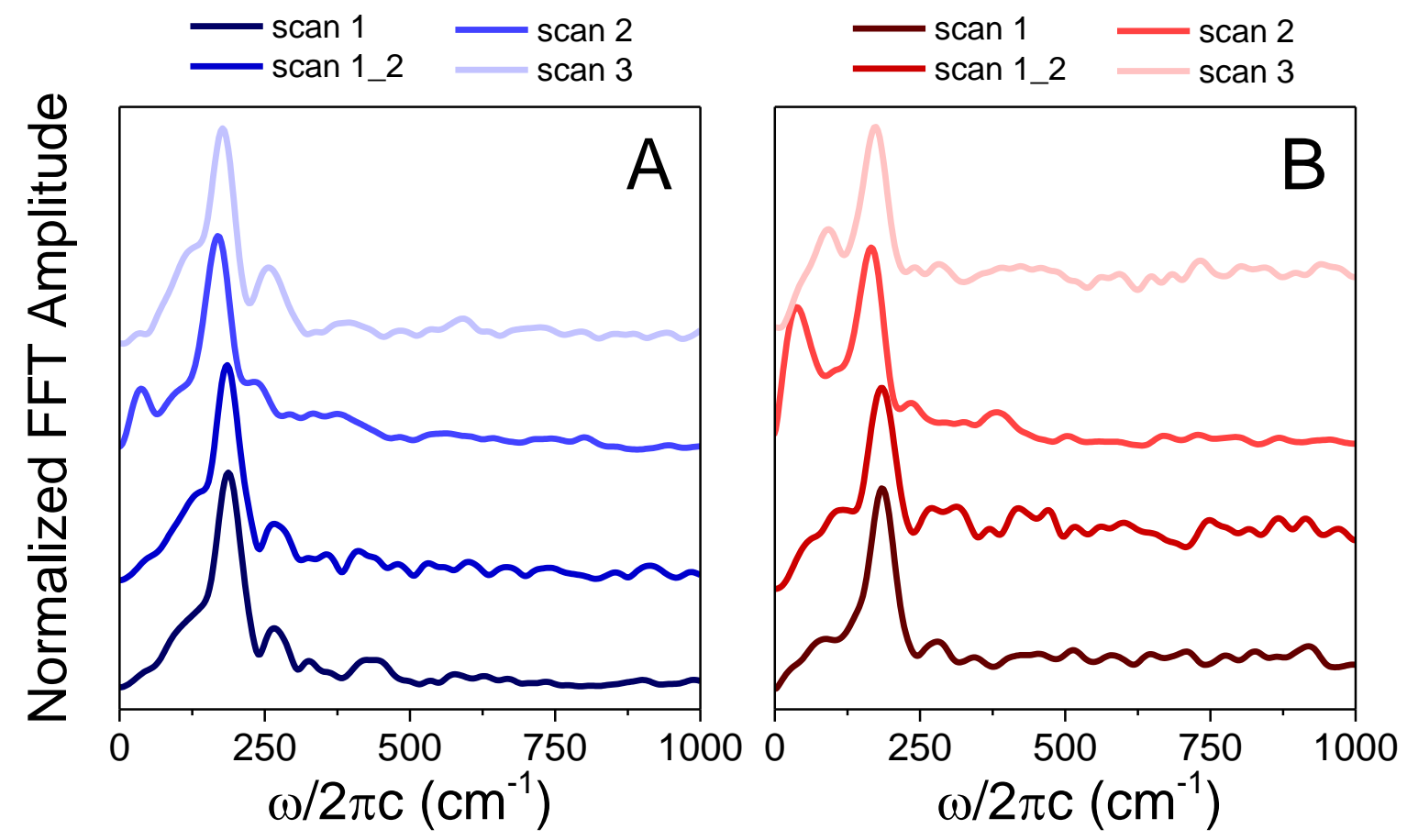

Figure S11. Normalized replicate Frobenius norms calculated across the $\boldsymbol{m}$-PDI (A) GSB/SE and (B) ESA spectral features. All scans were collected on separate days, except scans 1 and 1_2 which were performed on the same day, but with different sample batches.
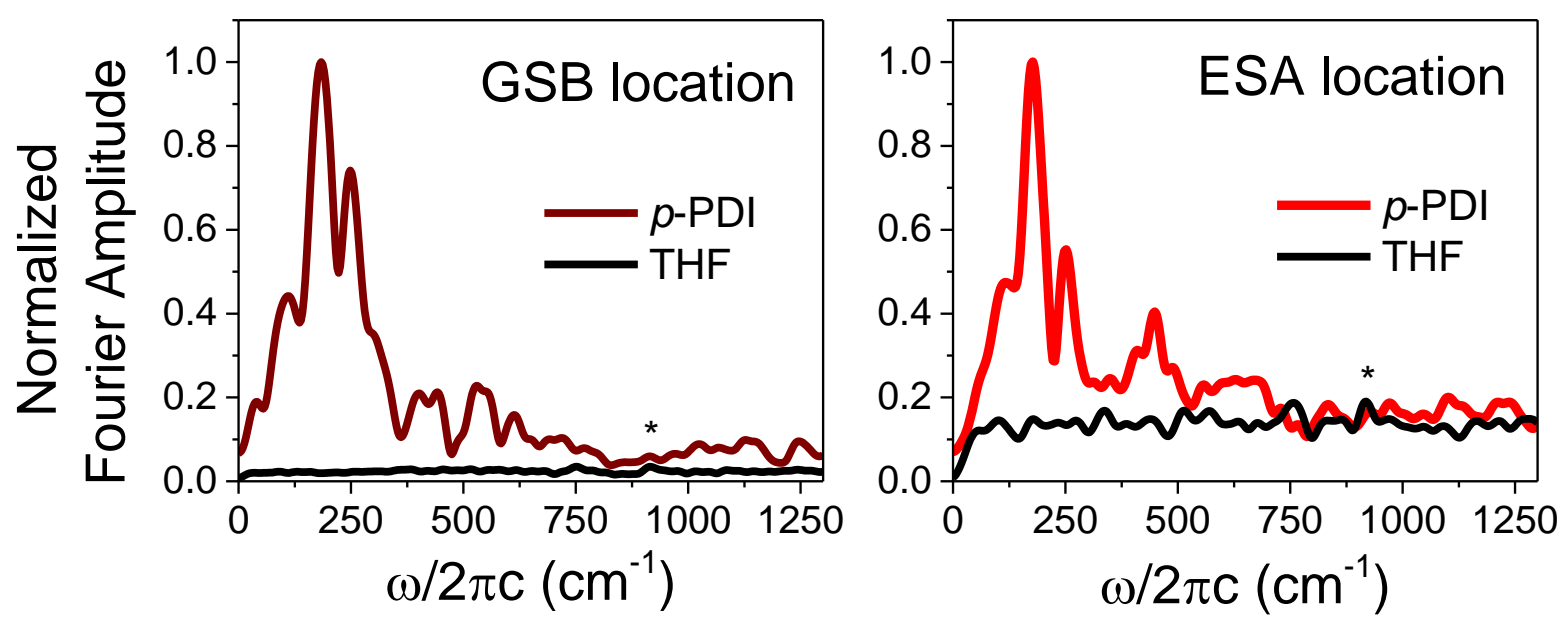

Figure S12. Frobenius norms calculated across the $\boldsymbol{p}$-PDI (A) GSB/SE and (B) ESA region for the both $\boldsymbol{p}$-PDI and the corresponding neat THF solvent scan. The power spectra are normalized to the maximum Fourier magnitude in the respective $\boldsymbol{p}$-PDI power spectrum. 
To investigate any influence the benzyl linker has on the power spectra in these studies, we conducted identical 2DES experiments on a monomeric compound with propyl groups on the imide positions. A direct comparison of excited-state power spectra for the monomeric compounds is shown in Figure S13; we observe no significant differences.

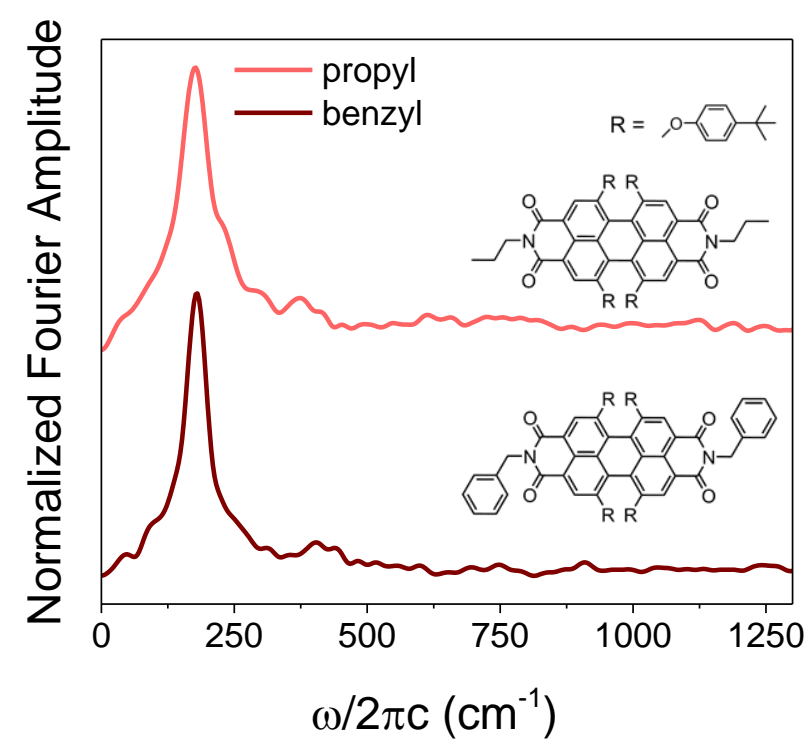

Figure S13. ESA Frobenius norms for the monomeric PDI control compounds.

\section{Quantum beating maps}

To further confirm that the weaker quantum beating signatures around $400 \mathrm{~cm}^{-1}$ in these compounds was not an artifact of noise, we produced beating amplitude maps for PDI-Ref and $\boldsymbol{p}$-PDI, as shown in Figure S14. For both samples, the amplitude of this frequency does not simply follow the lineshapes of the GSB and SE optical features. Instead, the peaks in the beatmaps occur at positions expected for a vibrational coherence, or vibronic in the case of the dimer. It should be noted that drawing insight about vibronic coupling from these beatmaps is complicated by the significant Stokes shift in these systems (vide infra), which results in the excited-state coherence 
amplitude smearing in the probe dimension below the diagonal. This notion is further supported by the presence of strong quantum beating near the fluorescence maximum in both compounds.
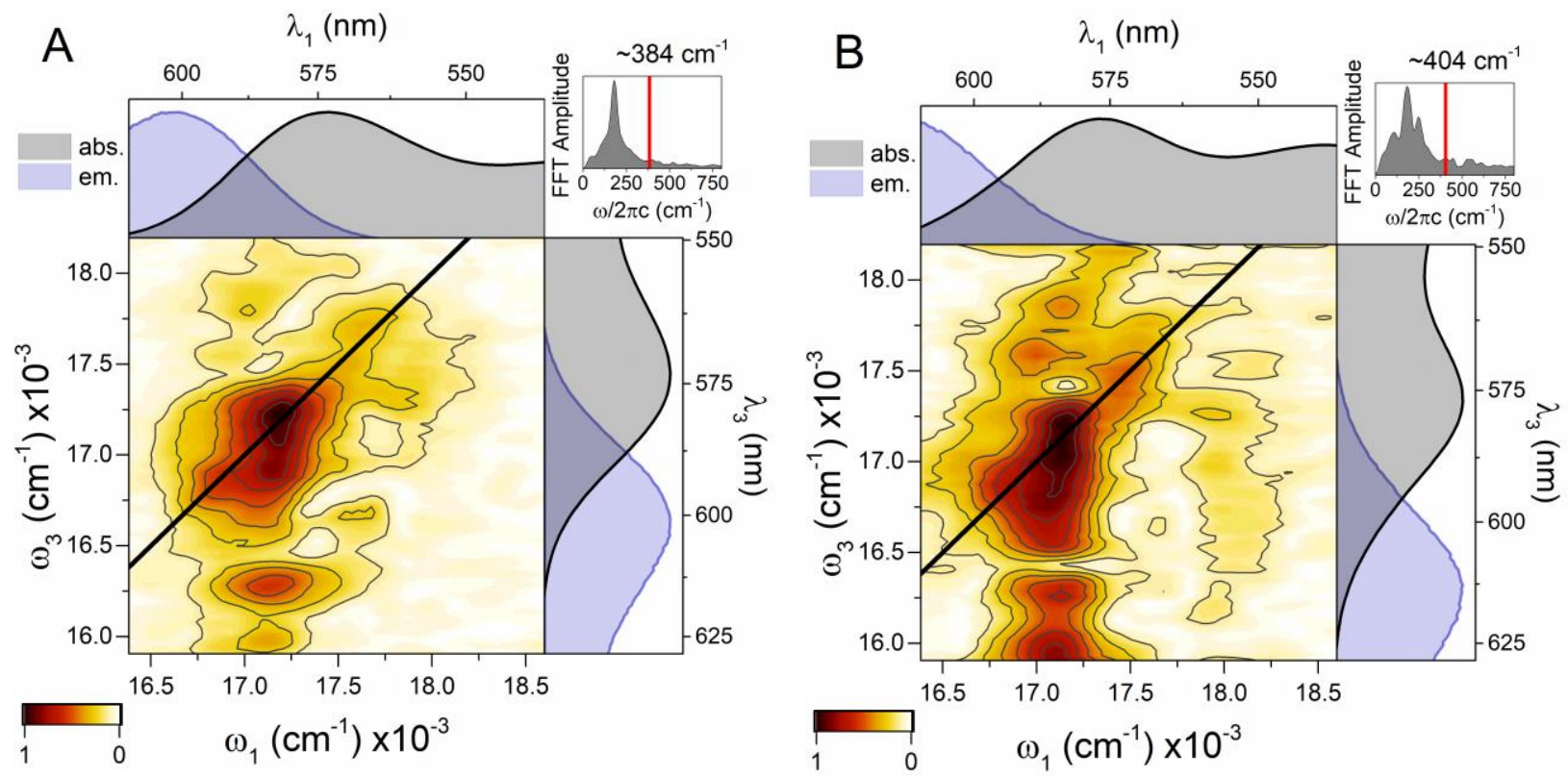

Figure S14. Quantum beating amplitude maps at a frequency near $400 \mathrm{~cm}^{-1}$ for (A) PDI-Ref and (B) $\boldsymbol{p}$-PDI. Absorption and emission spectra for both compounds are included.

\section{Impact of resonance condition and high-frequency coherences}

To investigate influence from the resonance conditions employed for the main text experiments and any differences in high-frequency coherences in these systems, we conducted further experiments with our 2DES pump pulse centered near $18000 \mathrm{~cm}^{-1}(\sim 555 \mathrm{~nm})$ to enforce more resonance with higher-lying vibronic states. This pump spanned 17000 to $19100 \mathrm{~cm}^{-1}$ with a $\sim 940 \mathrm{~cm}^{-1}$ FWHM. Figure S15 shows the spectra of the pulses employed as well as SHG-FROG characterization of the pump. All samples were prepared as described in section 1.2. 

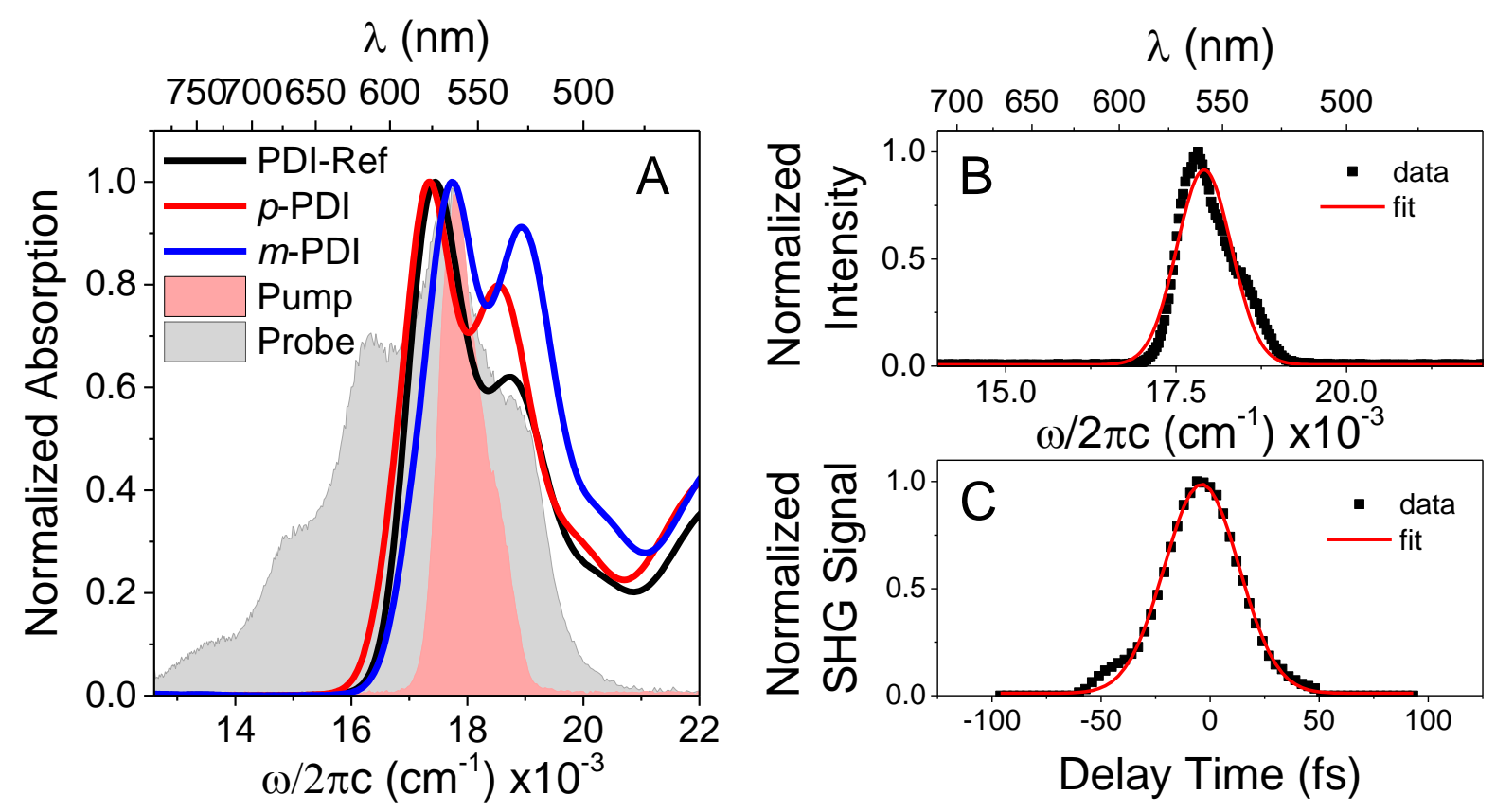

Figure S15. (A) Normalized steady-state absorption spectra of PDI-Ref, $\boldsymbol{p}$-PDI, and $\boldsymbol{m}$-PDI obtained in THF at room temperature. Pump and probe pulses for the 2DES measurements are superimposed. (B) Raw pump spectrum fit to a Gaussian $\left(\sim 940 \mathrm{~cm}^{-1} \mathrm{FWHM}\right)$ and (C) a SHGFROG summed across all wavelengths and fit to a Gaussian ( $\sim 40$ fs FWHM, corresponding to $\mathrm{a} \sim 28$ fs pulse duration).

An identical procedure as described for the $\sim 17540 \mathrm{~cm}^{-1}(\sim 570 \mathrm{~nm})$ 2DES pump experiments was used to obtain excited- and primarily ground-state power spectra for each compound, as shown in Figure S16. As mentioned in the main text, the excited-state signals cannot be completely removed from the latter. Despite increased resonance with any higher-lying vibronic states, no appreciable peaks can be observed above $\sim 1000 \mathrm{~cm}^{-1}$, with the exception of the weak peak near $\sim 1500 \mathrm{~cm}^{-1}$. All low frequency results for this experiment agree with the trends discussed in the main text and corroborate the relative enhancement of certain quantum beating signals from the $\boldsymbol{p}$-PDI compound in relation to the other samples. Figure S17 illustrates the magnitude of these beating signals from $\boldsymbol{p}$-PDI in comparison to that obtained from the corresponding spectral regions of a neat-THF 2DES blank scan. 


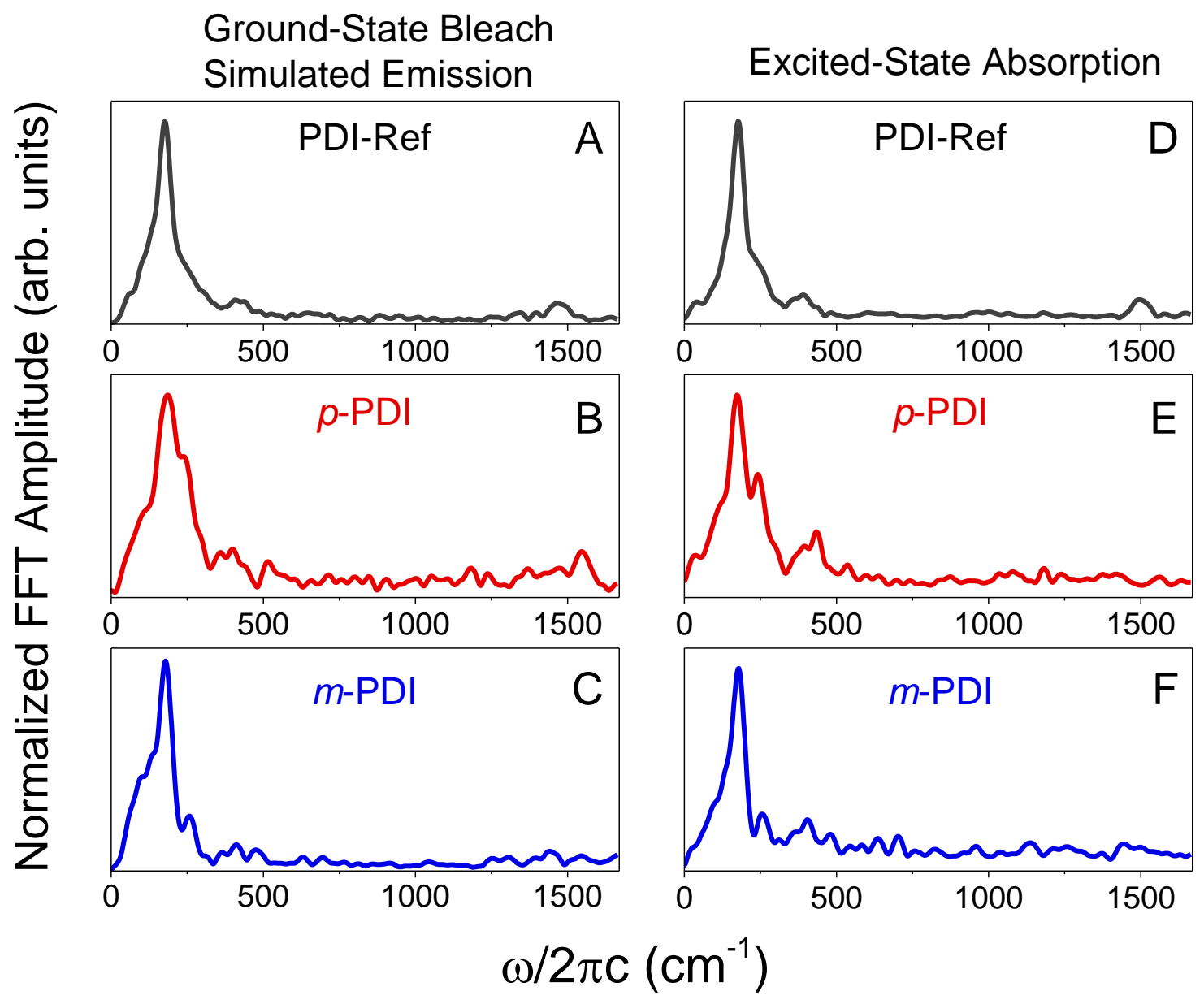

Figure S16. Frobenius norms calculated in the frequency domain across a $300 \mathrm{x} 400 \mathrm{~cm}^{-1}$ region on the (A-C) GSB/SE feature near the diagonal and (D-F) the higher energy $\mathrm{S}_{\mathrm{n}} \leftarrow \mathrm{S}_{1}$ ESA feature for each system listed. 

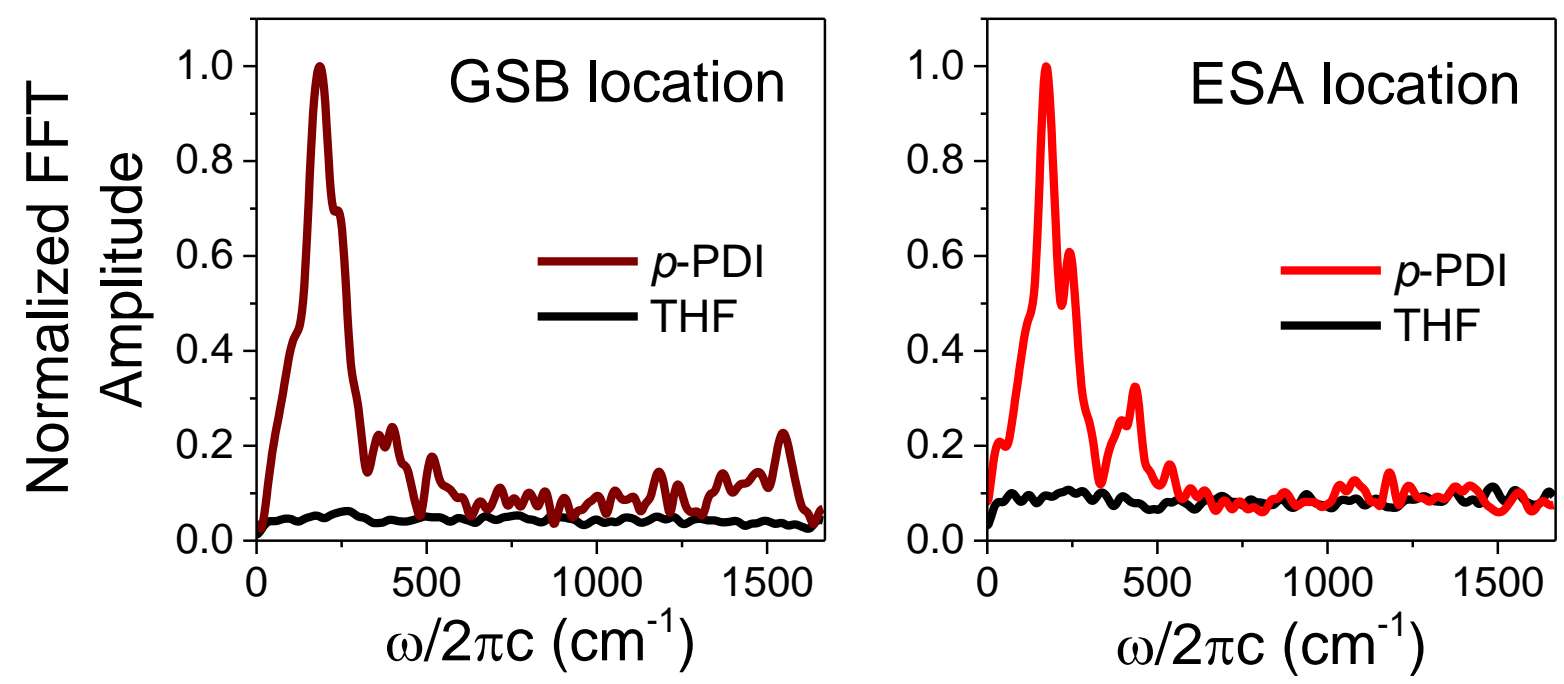

Figure S17. Frobenius norms calculated across the $\boldsymbol{p}$-PDI (A) GSB/SE and (B) ESA region for the both $\boldsymbol{p}$-PDI and neat THF pumped at $18000 \mathrm{~cm}^{-1}$. The power spectra are normalized to the maximum Fourier magnitude in the respective $\boldsymbol{p}$-PDI power spectra.

\section{Frobenius norm region selection}

Frobenius norms are used here to avoid bias in selecting a region of the $2 \mathrm{D}$ spectrum to analyze coherences as well as for improving the signal-to-noise ratio. All frequency-domain Frobenius norms span 400 and $300 \mathrm{~cm}^{-1}$ in the pump and probe dimensions, respectively, and cover $\sim 350$ points in the 2D maps. These regions were selected to be located near the maximum beating amplitude of the $\sim 430 \mathrm{~cm}^{-1}$ oscillation since a signature near this frequency was observed in all of these compounds, whereas the common $\sim 180 \mathrm{~cm}^{-1}$ oscillation shows very broad features in the frequency amplitude domain and complicates selection of the center. In cases where the signal-tonoise ratio for this mode was too low, we centered the Frobenius norm near the $\sim 250 \mathrm{~cm}^{-1}$ feature amplitude maximum. In principle, all of these coherence frequencies should have similar maximum amplitude coordinates within data for each respective compound. Figure S18 illustrates representative time- and frequency-domain spectra for $\boldsymbol{p}$-PDI with the norm regions superimposed. Similar Frobenius norms conducted in the time domain are shown in Figure 3A-C, but these traces 
were constructed over a region containing only $\sim 18$ points (centered at the specified coordinates) to avoid destructive interference of the time-domain oscillations.
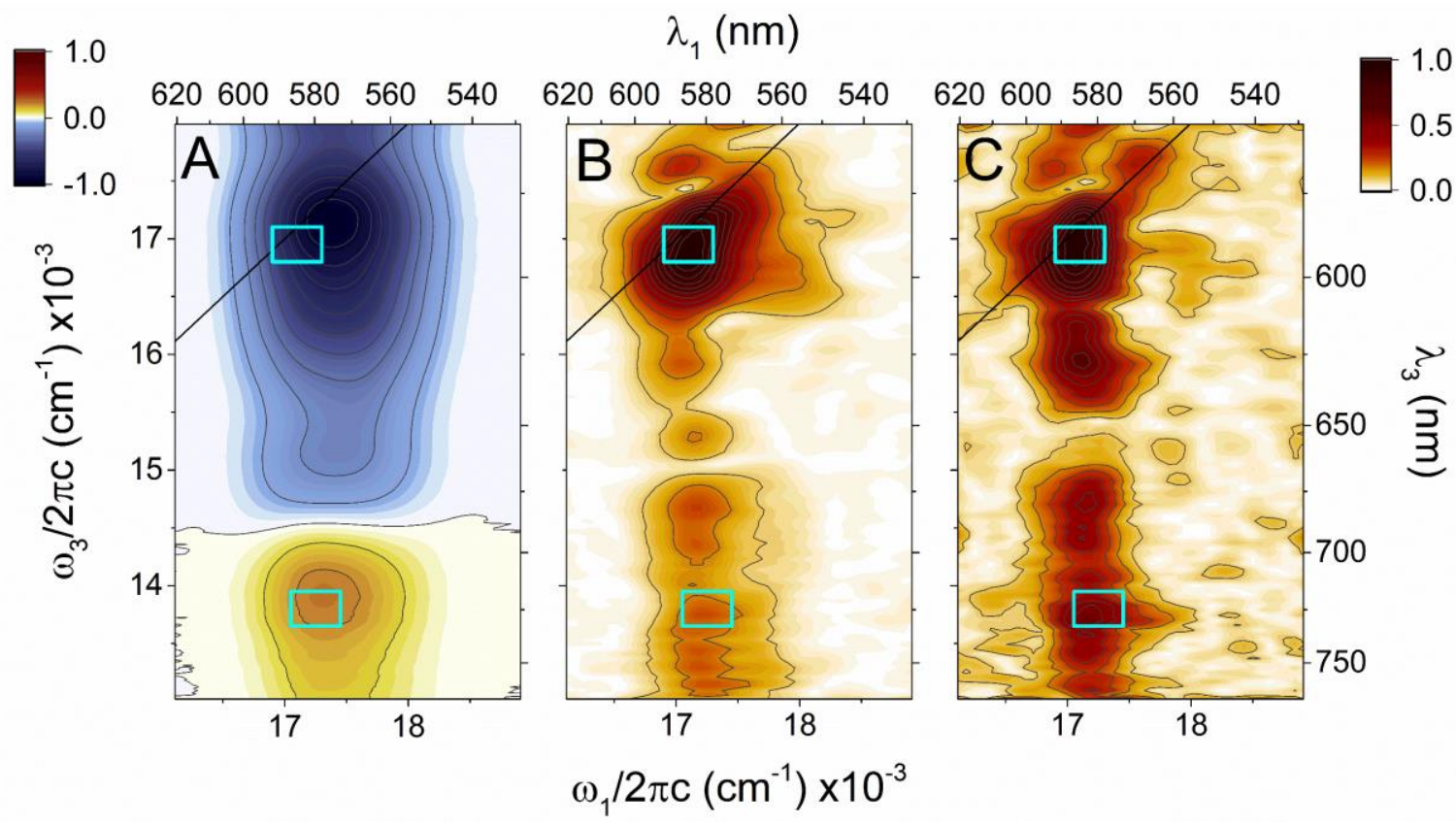

Figure S18. (A) Time-domain and (B and C) frequency-domain spectra illustrating region selection for Frobenius norms for $\boldsymbol{p}$-PDI. The frequency amplitude maps reflect the magnitude of oscillatory signals near (B) 257 and (C) $439 \mathrm{~cm}^{-1}$. Cyan rectangles indicate the approximate regions over which the coherence analysis was performed.

\section{Characterization of influence from the dynamic Stokes shift}

Figure S19 shows a closer comparison of the absorption and fluorescence spectra for PDIRef, $\boldsymbol{p}$-PDI, and $\boldsymbol{m}$-PDI. Stokes shifts of $\sim 850, \sim 1000$, and $\sim 1200 \mathrm{~cm}^{-1}$, respectively, are observed. In order to understand how the coherences on the ground versus excited state will manifest themselves in the frequency-domain beatmaps due to this shift, we characterized the timescale of the dynamic Stokes shift in PDI-Ref. Considering that the excited-state coherences stem from coherently produced wavepackets, theory predicts a $\pi$ phase flip where the wavepacket traverses the minimum of the excited-state potential energy surface. ${ }^{7,} 10$ This node in the signal can be used 
to characterize the timescale of the Stokes shift. ${ }^{6,11}$ Figure S19D shows the oscillatory residuals for PDI-Ref after removing the population dynamics from a transient absorption dataset using a function containing two exponential terms convoluted with a Gaussian. The phase jump and amplitude node for the intense $\sim 180 \mathrm{~cm}^{-1}$ mode is clearly seen near $16500 \mathrm{~cm}^{-1}(607 \mathrm{~nm})$. However, at the earliest time point shown, the node is located near $16950 \mathrm{~cm}^{-1}(590 \mathrm{~nm})$, which is the initial vertical energy between the ground state and the displaced excited state. The node transiently shifts and arrives at its final position after $\sim 150 \mathrm{fs}$. This shift directly represents the timescale of the dynamics Stokes shift for PDI-Ref in THF at room temperature. The origin of this rapid shift is likely inertial solvent dynamics. ${ }^{6,11}$ In the dimers, this is convoluted with relaxation between exciton states. ${ }^{8}$ 

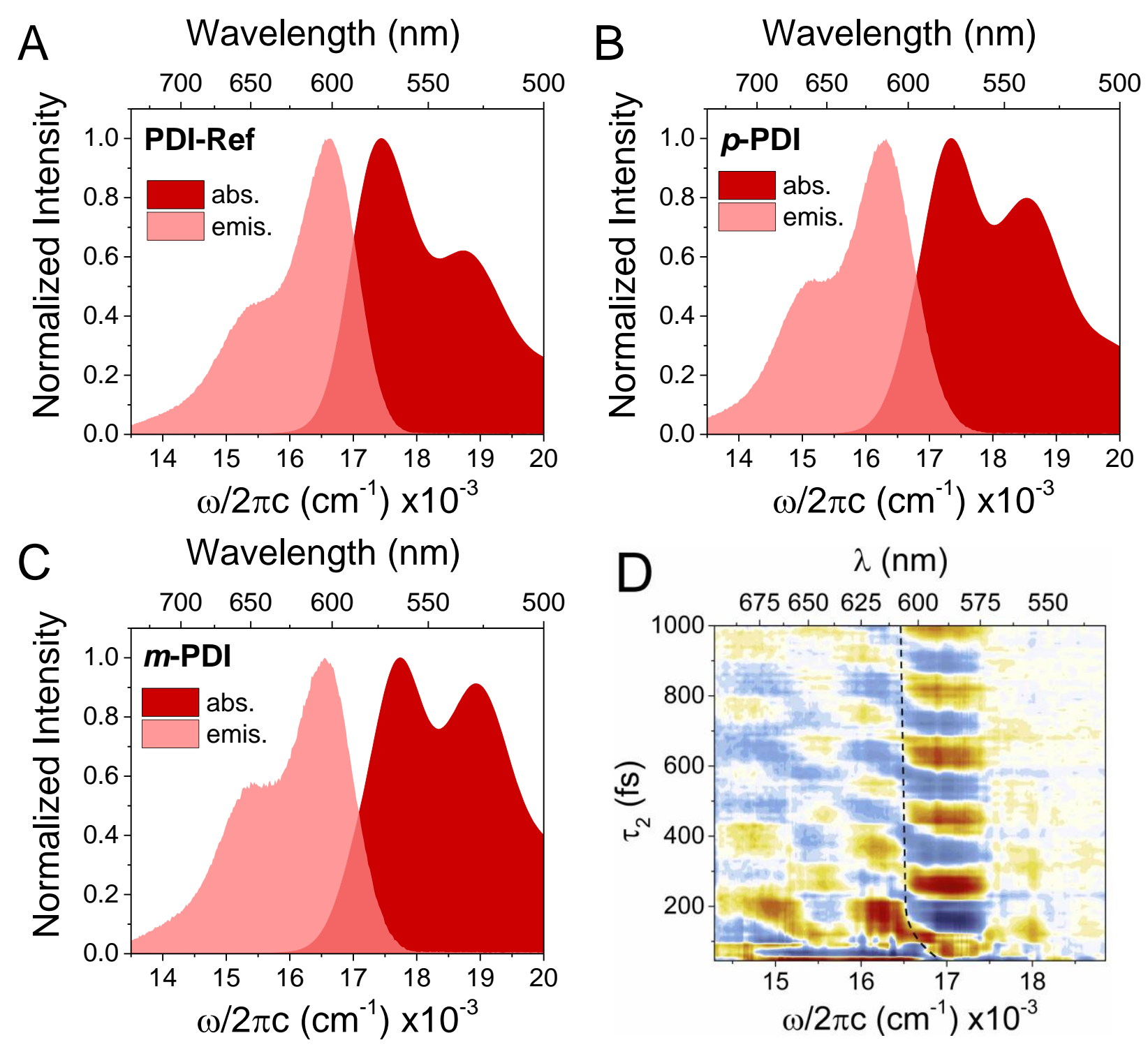

Figure S19. Comparison of absorption and fluorescence spectra for (A) PDI-Ref, (B) p-PDI, and (C) $\boldsymbol{m}$-PDI in THF at room temperature and (D) residuals from a transient absorption scan of PDI-Ref post removal of population dynamics. The superimposed dotted line illustrates the approximate location of the amplitude node in the oscillatory signals.

\section{Additional femtosecond stimulated Raman spectroscopy data}

\subsection{Additional details}

Calibration of the CCD array for all FSRS data was performed with at least four neat solvent peaks (DCM, DMF, and THF) and the Rayleigh line. The ground-state FSRS spectra presented in Figure 4D-F were obtained via dispersing onto the CCD array with a $2400 \mathrm{gr} / \mathrm{mm}$ 
grating and averaging 100 one-second exposures. Broad optical absorption baselines were removed via a cubic fit. The spectra were smoothed with adjacent averaging over a 7-point range for clarity.

\subsection{Additional spectra}

Figure S20 compares cubic baseline-subtracted, unsmoothed, and unnormalized FSRS spectra for each compound compared to that of neat-THF.

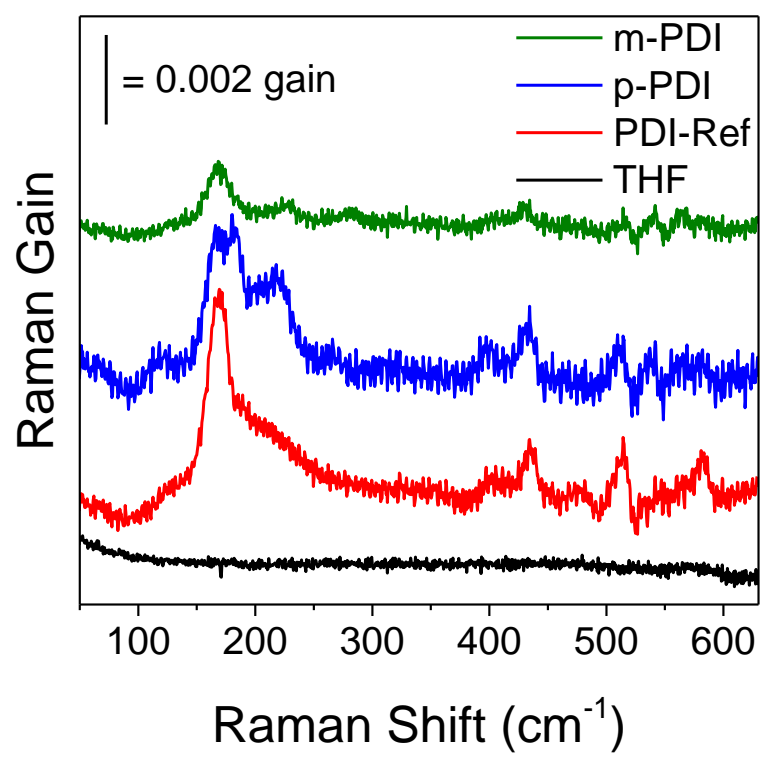

Figure S20. Unnormalized and unsmoothed FSRS spectra for PDI-Ref, $\boldsymbol{p}$-PDI, and $\boldsymbol{m}$-PDI in THF at room temperature compared to that of neat-THF.

We conducted excited-state time-resolved FSRS on all three of the compounds studied here to complement the power spectra extracted from the 2DES ESA features (Figure 3D-F). Figure S21 shows FSRS spectra $0.5 \mathrm{ps}$ after photoexcitation via a $573 \mathrm{~nm}$ actinic pump. The $710 \mathrm{~nm}$ Raman pump here minimizes resonance with stimulated emission while maximizing that with the higher energy $S_{n} \leftarrow S_{1}$ transition in these compounds. Raw data were collected using a $1200 \mathrm{gr} / \mathrm{mm}$ grating. The spectra in Figure S21 were each generated by averaging 100 one-second exposures, subtracting the corresponding -5 ps trace, removing the broad transient absorption background 
with a spline fit, and adjacent averaging over a 3-point range for clarity. Despite the lower signalto-noise ratio, the peak trends between PDI-Ref, $\boldsymbol{p}$-PDI, and $\boldsymbol{m}$-PDI mostly follow those observed in the 2DES ESA power spectra (Figure 3D-F).

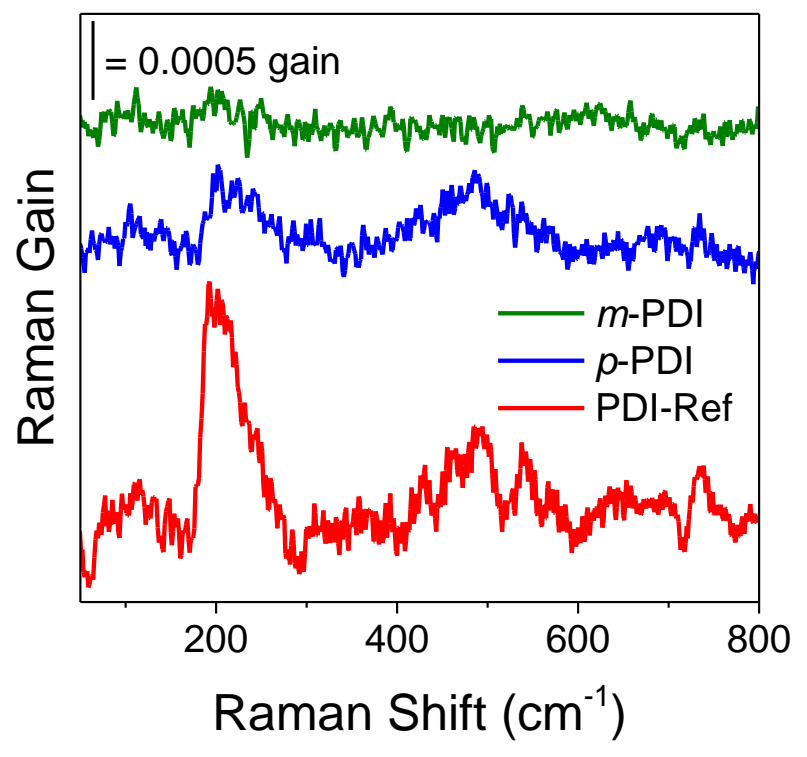

Figure S21. Unnormalized time-resolved FSRS spectra for PDI-Ref, $\boldsymbol{p}$-PDI, and $\boldsymbol{m}$-PDI in THF at room temperature $0.5 \mathrm{ps}$ after photoexcitation.

\section{Normal mode analysis}

Figure S22A illustrates the computed Raman frequency spectrum from the PDI-Ref DFT normal mode analysis. The spectrum was broadened (Gaussian FWHM $=10 \mathrm{~cm}^{-1}$ ) for ease of comparison to the experimental data and the frequency axis is scaled in accordance with our choice of basis set (x0.96). Figure S22B-D provide further depictions of the nuclear motions potentially associated with the peaks observed via 2DES and FSRS. However, the lack of inclusion of the resonance condition in these calculations complicates precise assignment of these modes. 

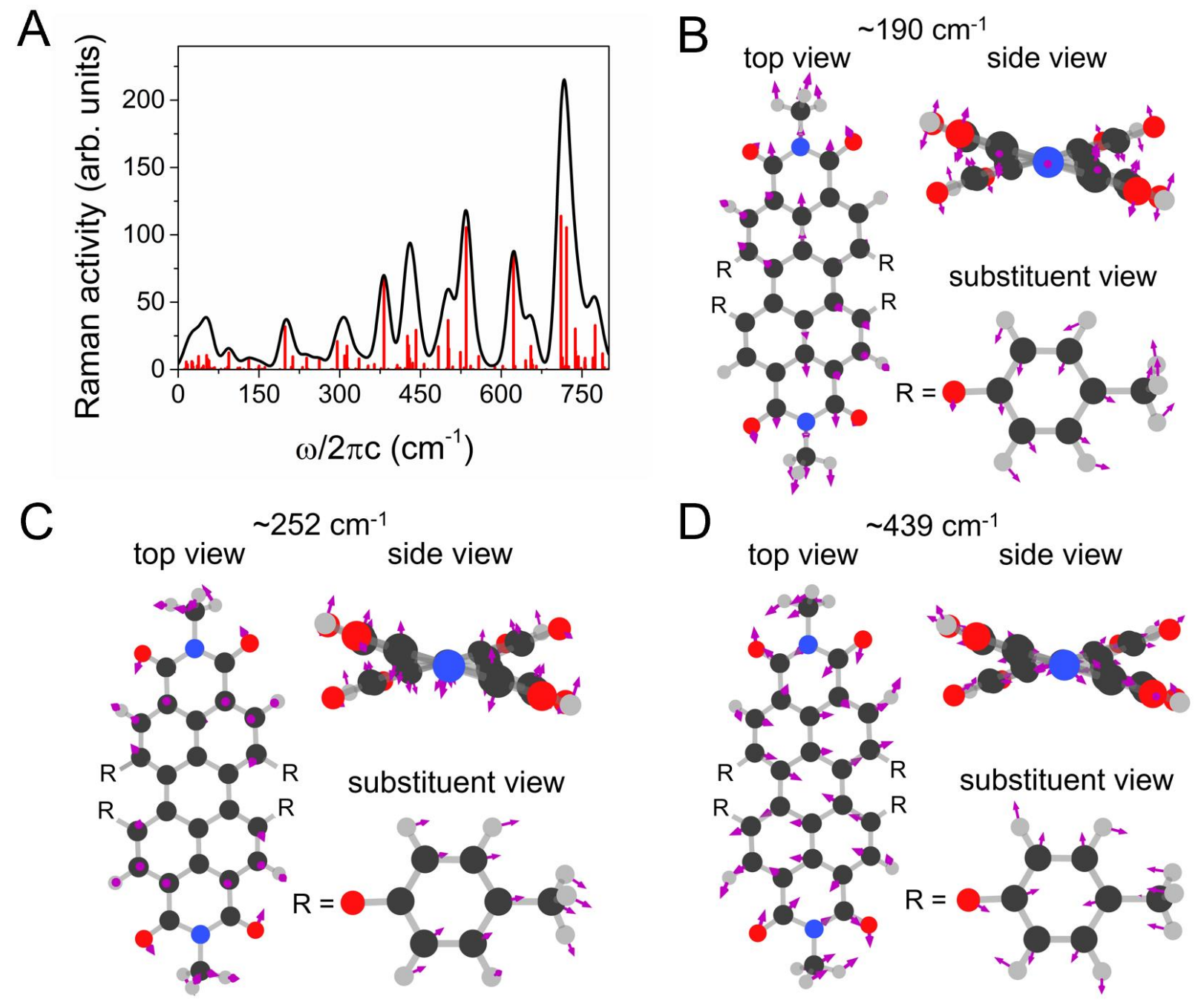

Figure S22. (A) Computed Raman spectrum for PDI-Ref and nuclear motions associated with vibrational frequencies of (B) $\sim 190$, (C) $\sim 252$, and (D) $\sim 439 \mathrm{~cm}^{-1}$ (scaled by 0.96).

\section{Computed Values}

System: PDI-Ref

Simplifications: methyl on phenoxy group instead of tert-butyl

\section{Coordinates:}

\begin{tabular}{l|llll|lll}
$\mathrm{O}$ & 2.16803 & -5.6741 & -0.70312 & $\mathrm{O}$ & 3.43219 & 0.73686 & 1.06366 \\
$\mathrm{O}$ & -2.19034 & -5.67213 & 0.67877 & $\mathrm{O}$ & 3.42485 & -0.75684 & -1.08777 \\
$\mathrm{O}$ & 2.19415 & 5.67703 & 0.6616 & $\mathrm{~N}$ & -0.01821 & -5.67232 & -0.00926 \\
$\mathrm{O}$ & -2.16824 & 5.67678 & -0.70759 & $\mathrm{~N}$ & 0.02012 & 5.6761 & -0.02042 \\
$\mathrm{O}$ & -3.42461 & 0.75858 & -1.08321 & $\mathrm{C}$ & 2.30638 & -2.85939 & -0.70644 \\
$\mathrm{O}$ & -3.42923 & -0.73202 & 1.07166 & $\mathrm{C}$ & 2.34446 & -1.45581 & -0.62459
\end{tabular}




\begin{tabular}{|c|c|c|c|c|c|c|c|}
\hline $\mathrm{C}$ & 1.23678 & -0.71922 & -0.15757 & $\mathrm{C}$ & 6.56737 & -1.87653 & -2.59304 \\
\hline $\mathrm{C}$ & -0.004 & -1.41988 & -0.00723 & $\mathrm{C}$ & -6.55397 & -1.85336 & 2.61416 \\
\hline $\mathrm{C}$ & -0.00879 & -2.84321 & -0.00712 & $\mathrm{C}$ & -5.27349 & -1.32059 & 2.46072 \\
\hline $\mathrm{C}$ & 1.16486 & -3.54984 & -0.34993 & $\mathrm{C}$ & -8.63061 & 3.02183 & -1.65555 \\
\hline $\mathrm{C}$ & -1.24019 & -0.71089 & 0.14285 & $\mathrm{C}$ & 8.62607 & 3.00034 & 1.73512 \\
\hline $\mathrm{C}$ & -2.35331 & -1.43907 & 0.61051 & $\mathrm{C}$ & -8.61195 & -3.03026 & 1.71444 \\
\hline $\mathrm{C}$ & -2.32501 & -2.84297 & 0.69225 & $\mathrm{C}$ & 8.61262 & -3.05903 & -1.67117 \\
\hline $\mathrm{C}$ & -1.1879 & -3.53994 & 0.33551 & $\mathrm{H}$ & 3.17183 & -3.41862 & -1.0392 \\
\hline $\mathrm{C}$ & -1.20376 & -5.02052 & 0.35997 & $\mathrm{H}$ & -3.1942 & -3.39717 & 1.02387 \\
\hline $\mathrm{C}$ & 1.17712 & -5.03209 & -0.37909 & $\mathrm{H}$ & 3.19865 & 3.40288 & 1.00917 \\
\hline $\mathrm{C}$ & 1.24197 & 0.71498 & 0.13905 & $\mathrm{H}$ & -3.17284 & 3.42047 & -1.03842 \\
\hline $\mathrm{C}$ & 0.00557 & 1.42372 & -0.00984 & $\mathrm{H}$ & 0.83093 & 7.49339 & -0.70474 \\
\hline $\mathrm{C}$ & -1.23548 & 0.72282 & -0.15637 & $\mathrm{H}$ & -0.92136 & 7.49926 & -0.32524 \\
\hline C & 2.3561 & 1.44387 & 0.60321 & $\mathrm{H}$ & -0.82991 & -7.49137 & -0.68799 \\
\hline $\mathrm{C}$ & 2.32845 & 2.84798 & 0.68138 & $\mathrm{H}$ & 0.92307 & -7.49596 & -0.31174 \\
\hline $\mathrm{C}$ & 1.19045 & 3.54425 & 0.32587 & $\mathrm{H}$ & -7.10221 & 2.8777 & 0.60784 \\
\hline $\mathrm{C}$ & 0.01035 & 2.84705 & -0.01239 & $\mathrm{H}$ & -7.05568 & 1.83702 & -3.55902 \\
\hline $\mathrm{C}$ & -1.16415 & 3.55309 & -0.35365 & $\mathrm{H}$ & -4.76409 & 0.89958 & -3.29148 \\
\hline $\mathrm{C}$ & -2.30648 & 2.86204 & -0.70656 & $\mathrm{H}$ & 6.99522 & 1.8697 & 3.61513 \\
\hline $\mathrm{C}$ & -2.34401 & 1.45859 & -0.62261 & $\mathrm{H}$ & 7.14294 & 2.84953 & -0.56384 \\
\hline C & 1.20663 & 5.02491 & 0.34688 & $\mathrm{H}$ & 4.88136 & 1.87436 & -0.87263 \\
\hline $\mathrm{C}$ & -1.1764 & 5.03531 & -0.38537 & $\mathrm{H}$ & -4.86533 & -1.87483 & -0.87068 \\
\hline $\mathrm{C}$ & 0.05833 & 7.14176 & -0.0165 & $\mathrm{H}$ & -7.13088 & -2.84163 & -0.57789 \\
\hline $\mathrm{C}$ & -0.05612 & -7.13797 & -0.00198 & $\mathrm{H}$ & 7.09996 & -2.88296 & 0.60093 \\
\hline C & -4.66886 & 1.37076 & -1.20228 & $\mathrm{H}$ & 4.8329 & -1.91318 & 0.869 \\
\hline $\mathrm{C}$ & -5.31877 & 1.92206 & -0.0966 & $\mathrm{H}$ & 4.76184 & -0.90325 & -3.29724 \\
\hline $\mathrm{C}$ & -6.59656 & 2.45154 & -0.25565 & $\mathrm{H}$ & 7.04795 & -1.852 & -3.56814 \\
\hline C & -7.24735 & 2.43388 & -1.49874 & $\mathrm{H}$ & 4.71789 & 0.91425 & 3.30282 \\
\hline $\mathrm{C}$ & -6.57317 & 1.86558 & -2.58508 & $\mathrm{H}$ & -4.8302 & 1.92057 & 0.87225 \\
\hline $\mathrm{C}$ & -5.28801 & 1.3379 & -2.44772 & $\mathrm{H}$ & -7.02165 & -1.83307 & 3.59564 \\
\hline $\mathrm{C}$ & 4.67496 & 1.34879 & 1.2026 & $\mathrm{H}$ & -4.73815 & -0.88587 & 3.29916 \\
\hline C & 5.26375 & 1.33775 & 2.4654 & $\mathrm{H}$ & -0.29852 & -7.49675 & 1.00138 \\
\hline $\mathrm{C}$ & 6.53951 & 1.87531 & 2.62752 & $\mathrm{H}$ & 0.30265 & 7.50282 & 0.98558 \\
\hline $\mathrm{C}$ & 7.23915 & 2.43027 & 1.54721 & $\mathrm{H}$ & 8.94145 & 3.57954 & 0.86162 \\
\hline $\mathrm{C}$ & 6.62027 & 2.42682 & 0.29072 & $\mathrm{H}$ & 9.36828 & 2.20611 & 1.88923 \\
\hline $\mathrm{C}$ & 5.34798 & 1.88613 & 0.10688 & $\mathrm{H}$ & 8.67381 & 3.65939 & 2.60978 \\
\hline $\mathrm{C}$ & -7.24226 & -2.41823 & 1.53376 & $\mathrm{H}$ & 9.13311 & -2.64659 & -2.54164 \\
\hline $\mathrm{C}$ & -4.6727 & -1.34358 & 1.2053 & $\mathrm{H}$ & 8.55025 & -4.14632 & -1.81191 \\
\hline C & -5.3384 & -1.88601 & 0.10575 & $\mathrm{H}$ & 9.23793 & -2.88325 & -0.78883 \\
\hline $\mathrm{C}$ & -6.61262 & -2.42107 & 0.28072 & $\mathrm{H}$ & -9.07593 & 2.74122 & -2.61514 \\
\hline $\mathrm{C}$ & 7.24049 & -2.44787 & -1.5068 & $\mathrm{H}$ & -8.60765 & 4.11842 & -1.61026 \\
\hline $\mathrm{C}$ & 6.59388 & -2.4568 & -0.26222 & $\mathrm{H}$ & -9.3034 & 2.68098 & -0.85992 \\
\hline $\mathrm{C}$ & 5.31859 & -1.92039 & -0.10126 & $\mathrm{H}$ & -9.23221 & -2.89405 & 0.82185 \\
\hline $\mathrm{C}$ & 4.66817 & -1.37095 & -1.20683 & $\mathrm{H}$ & -9.14054 & -2.58655 & 2.5646 \\
\hline $\mathrm{C}$ & 5.28553 & -1.34271 & -2.45392 & $\mathrm{H}$ & -8.54388 & -4.11048 & 1.90002 \\
\hline
\end{tabular}


Raman Frequencies

\begin{tabular}{|c|c|c|c|c|c|}
\hline $\begin{array}{l}\text { Frequency } \\
\qquad\left(\mathrm{cm}^{-1}\right)\end{array}$ & $\begin{array}{l}\text { Raman Activity } \\
\text { (arb. units) }\end{array}$ & $\begin{array}{l}241.82 \\
262.14\end{array}$ & $\begin{array}{l}0.156 \\
7.759\end{array}$ & $\begin{array}{l}548.16 \\
558.22\end{array}$ & $\begin{array}{l}0.183 \\
9.746\end{array}$ \\
\hline 11.79 & 0.356 & 283.9 & 0.064 & 570.21 & 0.108 \\
\hline 13.81 & 0.801 & 287.16 & 0.069 & 588.3 & 1.766 \\
\hline 15.04 & 5.642 & 295.52 & 20.763 & 602.98 & 2.481 \\
\hline 17.33 & 3.645 & 309.34 & 10.198 & 607.95 & 0.16 \\
\hline 25.41 & 5.986 & 312.18 & 2.438 & 622.87 & 83.77 \\
\hline 26.79 & 5.103 & 313.61 & 17.427 & 625.94 & 2.41 \\
\hline 28.03 & 1.852 & 316 & 0.768 & 645.47 & 6.694 \\
\hline 35.31 & 2.705 & 329.41 & 0.485 & 645.92 & 2.795 \\
\hline 37.77 & 9.793 & 333.19 & 0.175 & 655.08 & 17.233 \\
\hline 43.75 & 0.996 & 336.03 & 7.949 & 656.49 & 7.434 \\
\hline 46.93 & 2.744 & 351.58 & 0.488 & 657.91 & 3.571 \\
\hline 52.88 & 10.495 & 352.85 & 2.731 & 658.74 & 3.241 \\
\hline 54.67 & 7.681 & 364.35 & 3.864 & 665.29 & 2.562 \\
\hline 56.31 & 4.598 & 376.84 & 0.699 & 684.39 & 0.074 \\
\hline 57.2 & 6.49 & 382.26 & 67.775 & 711.05 & 113.84 \\
\hline 58.99 & 1.198 & 390.28 & 0.303 & 713.48 & 8.711 \\
\hline 62.47 & 0.447 & 405.04 & 0.72 & 718.28 & 1.782 \\
\hline 65.15 & 0.97 & 406.94 & 3.239 & 718.98 & 2.433 \\
\hline 67.69 & 1.373 & 412.14 & 1.09 & 721.4 & $105.41^{\prime}$ \\
\hline 79.36 & 0.299 & 421.44 & 0.769 & 725.22 & 2.115 \\
\hline 86.83 & 0.504 & 425.97 & 24.822 & 737.59 & 30.203 \\
\hline 91.67 & 1.785 & 427.78 & 11.996 & 738.21 & $13.66 ?$ \\
\hline 92.51 & 0.471 & 428.13 & 17.7 & 743.36 & 4.09 \\
\hline 94 & 12.377 & 428.48 & 7.697 & 743.42 & 9.629 \\
\hline 110.73 & 0.948 & 429.42 & 4.621 & 748.3 & 0.908 \\
\hline 114.59 & 1.21 & 430.07 & 8.062 & 755.15 & 6.135 \\
\hline 121.68 & 0.244 & 435.51 & 4.798 & 755.75 & 8.489 \\
\hline 131.13 & 6.835 & 441.75 & 29.197 & 756.38 & 2.771 \\
\hline 132.51 & 0.16 & 456.84 & 4.022 & 770.28 & 3.899 \\
\hline 135.5 & 0.019 & 463.2 & 0.238 & 770.56 & 8.706 \\
\hline 143.52 & 0.007 & 472.92 & 0.235 & 774.58 & 32.697 \\
\hline 149.09 & 1.247 & 483.36 & 16.976 & 788.18 & 11.744 \\
\hline 150.18 & 2.678 & 498.57 & 0.68 & 791.82 & 1.368 \\
\hline 155.47 & 0.3 & 501.47 & 36.474 & 797.41 & 0.144 \\
\hline 160.82 & 1.523 & 502.85 & 15.236 & 818.85 & 2.018 \\
\hline 188.99 & 0.093 & 509.95 & 2.001 & 823.46 & 14.248 \\
\hline 198.61 & 31.696 & 524.25 & 12.823 & 843.51 & 19.876 \\
\hline 210.08 & 1.714 & 530.92 & 1.255 & 843.69 & 16.007 \\
\hline 212.89 & 9.521 & 535.05 & 105.404 & 845.9 & 10.861 \\
\hline 230.83 & 1.663 & 542.4 & 0.456 & 846.59 & 0.249 \\
\hline 238.6 & 8.538 & 543.17 & 2.568 & 848.62 & 10.889 \\
\hline
\end{tabular}




\begin{tabular}{|c|c|c|c|c|c|}
\hline 849.2 & 0.511 & 1159.83 & 515.341 & 1452.81 & 50.157 \\
\hline 851.6 & 63.012 & 1165.84 & 3.913 & 1455.12 & 19.829 \\
\hline 857.1 & 0.201 & 1165.86 & 3.165 & 1457.53 & 172.82 \\
\hline 859.5 & 3.438 & 1201.11 & 2.019 & 1457.86 & 55.97 \\
\hline 863.52 & 135.148 & 1201.33 & 10.138 & 1459.82 & 1149.911 \\
\hline 888.74 & 25.707 & 1202.17 & 5.861 & 1460.33 & 36.941 \\
\hline 894.91 & 2.233 & 1203.33 & 97.345 & 1462.58 & 72.65 \\
\hline 915.45 & 7.835 & 1204.08 & 5.687 & 1471.74 & 3.067 \\
\hline 924.6 & 135.253 & 1222.41 & 85.447 & 1485.06 & 2132.431 \\
\hline 925.4 & 6.336 & 1223.12 & 1.195 & 1516.84 & 26.048 \\
\hline 925.66 & 61.844 & 1238.6 & 5.293 & 1517.64 & 25.713 \\
\hline 926.34 & 3.802 & 1238.9 & 3.437 & 1518.24 & 26.056 \\
\hline 957.12 & 4.998 & 1239.42 & 0.471 & 1519.13 & 27.725 \\
\hline 958.21 & 3.491 & 1239.93 & 92.277 & 1523.92 & 26.388 \\
\hline 959 & 1.626 & 1245 & 71.82 & 1523.96 & 23.345 \\
\hline 960.33 & 1.973 & 1247.89 & 1.417 & 1525.67 & 14.267 \\
\hline 965.44 & 1.171 & 1254.87 & 13.364 & 1525.78 & 25.937 \\
\hline 966.47 & 2.74 & 1258.71 & 45.757 & 1525.89 & 35.851 \\
\hline 966.6 & 0.785 & 1259.95 & 531.334 & 1526.93 & 23.785 \\
\hline 966.92 & 3.254 & 1280.5 & 379.672 & 1529.77 & 19.56 \\
\hline 997.54 & 95.046 & 1309.97 & 176.475 & 1529.81 & 134.659 \\
\hline 998.59 & 70.438 & 1317.22 & 26.738 & 1553.56 & 30.367 \\
\hline 1019.74 & 4.068 & 1319.46 & 415.373 & 1553.76 & 5.397 \\
\hline 1020.18 & 2.279 & 1324.51 & 35.487 & 1555.4 & 5.324 \\
\hline 1021.39 & 2.253 & 1337.18 & 10.742 & 1559.86 & 175.179 \\
\hline 1021.71 & 2.711 & 1338.51 & 347.091 & 1561.42 & 36.756 \\
\hline 1022.5 & 3.274 & 1339.29 & 5.188 & 1561.91 & 2.96 \\
\hline 1039.57 & 4.431 & 1339.45 & 6.45 & 1581.21 & 7853.347 \\
\hline 1039.94 & 1.641 & 1348.54 & 18.006 & 1605.52 & 3.649 \\
\hline 1040.21 & 9.566 & 1348.97 & 12.852 & 1632.19 & 51.564 \\
\hline 1040.6 & 1.65 & 1349.11 & 82.654 & 1633.22 & 72.85 \\
\hline 1043.83 & 26.067 & 1349.6 & 37.873 & 1641.41 & 2232.713 \\
\hline 1066.41 & 0.145 & 1350.82 & 9.741 & 1641.92 & 2843.171 \\
\hline 1076.18 & 1.044 & 1366.13 & 314.704 & 1643.53 & 70.546 \\
\hline 1076.21 & 1.098 & 1372.88 & 8.001 & 1644.04 & 709.425 \\
\hline 1076.45 & 0.364 & 1382.9 & 8.158 & 1644.78 & 57.289 \\
\hline 1079.98 & 1.486 & 1384.81 & 9299.586 & 1649.87 & 1237.739 \\
\hline 1092.72 & 202.514 & 1392.88 & 270.085 & 1669.22 & 42.833 \\
\hline 1141.54 & 0.699 & 1423.89 & 743.917 & 1669.76 & 565.362 \\
\hline 1141.61 & 8.118 & 1434.06 & 12.837 & 1670.5 & 60.287 \\
\hline 1142.05 & 0.615 & 1445.02 & 52.112 & 1670.69 & 598.306 \\
\hline 1142.21 & 2.442 & 1445.85 & 43.832 & 1737.94 & 31.797 \\
\hline 1147.83 & 10.03 & 1446.3 & 99.504 & 1738.03 & 215.902 \\
\hline 1153.4 & 4.968 & 1447.05 & 81.834 & 1770.08 & 2.396 \\
\hline 1157.39 & 3.624 & 1449.62 & 37.341 & 1773.35 & 1514.181 \\
\hline
\end{tabular}




\begin{tabular}{c|cc|cc|c}
3042.6 & 254.888 & 3127.62 & 110.609 & 3217.08 & 73.95 \\
3043.19 & 123.445 & 3149.79 & 106.931 & 3217.82 & 80.94 \\
3043.22 & 520.902 & 3149.85 & 99.177 & 3218.13 & 56.405 \\
3043.98 & 357.377 & 3183.96 & 127.488 & 3218.17 & 72.382 \\
3087.49 & 75.188 & 3185.92 & 148.414 & 3221.85 & 65.662 \\
3087.56 & 510.651 & 3185.94 & 103.252 & 3221.9 & 78.078 \\
3097.7 & 112.61 & 3186.36 & 122.855 & 3222.42 & 34.104 \\
3097.97 & 102.102 & 3187.06 & 94.715 & 3222.71 & 40.026 \\
3099.99 & 100.135 & 3187.46 & 87.443 & 3249.48 & 79.754 \\
3100.72 & 101.974 & 3187.7 & 111.237 & 3249.57 & 114.413 \\
3126.85 & 62.223 & 3190.56 & 93.635 & 3249.85 & 91.019 \\
3127.19 & 79.735 & 3213.45 & 38.691 & 3250.25 & 97.696 \\
3127.56 & 29.793 & 3213.47 & 43.586 & &
\end{tabular}

\section{References for Supporting Information}

1. Spenst, P.; Würthner, F. A Perylene Bisimide Cyclophane as a "Turn-On" and "TurnOff' Fluorescence Probe. Angew. Chem. 2015, 54, 10165-10168.

2. $\quad$ Spenst, P.; Young, R. M.; Wasielewski, M. R.; Wurthner, F. Guest and solvent modulated photo-driven charge separation and triplet generation in a perylene bisimide cyclophane. Chem Sci 2016, 7, 5428-5434.

3. Mandal, A.; Chen, M.; Foszcz, E.; Schultz, J. D.; Kearns, N. M.; Young, R. M.; Zanni, M. T.; Wasielewski, M. R. Two-Dimensional Electronic Spectroscopy Reveals Excitation Energy-Dependent State Mixing during Singlet Fission in a Terrylenediimide Dimer. J Am Chem Soc 2018.

4. Middleton, C. T.; Woys, A. M.; Mukherjee, S. S.; Zanni, M. T. Residue-specific structural kinetics of proteins through the union of isotope labeling, mid-IR pulse shaping, and coherent 2D IR spectroscopy. Methods 2010, 52, 12-22.

5. Brown, K. E.; Veldkamp, B. S.; Co, D. T.; Wasielewski, M. R. Vibrational Dynamics of a Perylene-Perylenediimide Donor-Acceptor Dyad Probed with Femtosecond Stimulated Raman Spectroscopy. J Phys Chem Lett 2012, 3, 2362-6. 
6. Jumper, C. C.; Arpin, P. C.; Turner, D. B.; McClure, S. D.; Rafiq, S.; Dean, J. C.; Cina, J. A.; Kovac, P. A.; Mirkovic, T.; Scholes, G. D. Broad-Band Pump-Probe Spectroscopy Quantifies Ultrafast Solvation Dynamics of Proteins and Molecules. J. Phys. Chem. Lett. 2016, 7, 4722-4731.

7. Cina, J. A.; Kovac, P. A.; Jumper, C. C.; Dean, J. C.; Scholes, G. D. Ultrafast transient absorption revisited: Phase-flips, spectral fingers, and other dynamical features. J. Chem. Phys. 2016, $144,175102$.

8. Jumper, C. C.; Anna, J. M.; Stradomska, A.; Schins, J.; Myahkostupov, M.; Prusakova, V.; Oblinsky, D. G.; Castellano, F. N.; Knoester, J.; Scholes, G. D. Intramolecular radiationless transitions dominate exciton relaxation dynamics. Chem. Phys. Lett. 2014, 599, 23-33.

9. $\quad$ Halpin, A.; Johnson, P. J.; Tempelaar, R.; Murphy, R. S.; Knoester, J.; Jansen, T. L.; Miller, R. J. Two-dimensional spectroscopy of a molecular dimer unveils the effects of vibronic coupling on exciton coherences. Nat Chem 2014, 6, 196-201.

10. Pollard, W. T.; Dexheimer, S. L.; Wang, Q.; Peteanu, L. A.; Shank, C. V.; Mathies, R. A. Theory of dynamic absorption spectroscopy of nonstationary states. 4. Application to 12-fs resonant impulsive Raman spectroscopy of bacteriorhodopsin. J Phys Chem 1992, 96, 61476158.

11. McClure, S. D.; Turner, D. B.; Arpin, P. C.; Mirkovic, T.; Scholes, G. D. Coherent Oscillations in the PC577 Cryptophyte Antenna Occur in the Excited Electronic State. J. Phys. Chem. B 2014, 118, 1296-1308. 